\title{
Generalized orbifold Euler characteristics for general orbifolds and wreath products
}

\author{
CARLA FARSI \\ CHRISTOPHER SEATON
}

\begin{abstract}
We introduce the $\Gamma$-Euler-Satake characteristics of a general orbifold $Q$ presented by an orbifold groupoid $\mathcal{G}$, extending to orbifolds that are not global quotients the generalized orbifold Euler characteristics of Bryan-Fulman and Tamanoi. Each of these Euler characteristics is defined as the Euler-Satake characteristic of the space of $\Gamma$-sectors of the orbifold where $\Gamma$ is a finitely generated discrete group. We study the behavior of these Euler characteristics under product operations applied to the group $\Gamma$ as well as the orbifold and establish their relationships to existing Euler characteristics for orbifolds. As applications, we generalize formulas of Tamanoi, Wang and Zhou for the Euler characteristics and Hodge numbers of wreath symmetric products of global quotient orbifolds to the case of quotients by compact, connected Lie groups acting locally freely, in particular including all closed, effective orbifolds.
\end{abstract}

22A22, 55S15; 58E40, 55N91

\section{Introduction}

When Satake [19] first introduced orbifolds under the name $V$-manifolds, one of the first invariants defined was the Euler-Satake characteristic, then called the Euler characteristic as a $V$-manifold. Since that time, a number of Euler characteristics have been introduced for orbifolds. Most notably, the stringy orbifold Euler characteristic was introduced by Dixon, Harvey, Vafa and Witten in [7] for global quotients, ie quotients of manifolds by a finite group, and later generalized to general orbifolds by Roan in [17]; see also Atiyah and Segal [3] and Hirzebruch and Höfer [10]. This definition was generalized for global quotients first by Bryan and Fulman in [5] where it appeared as an element of a sequence of Euler characteristics, and independently by Tamanoi in $[21 ; 22]$ where an Euler characteristic was defined for each group $\Gamma$, the sequence of Bryan and Fulman corresponding to $\Gamma=\mathbb{Z}^{m}$.

Here, we extend these definitions to a general orbifold $Q$ presented by an orbifold groupoid $\mathcal{G}$, introducing for each finitely generated discrete group $\Gamma$ the $\Gamma-$ EulerSatake characteristic $\chi_{\Gamma}^{\mathrm{ES}}(Q)$. The $\Gamma$-Euler-Satake characteristic is defined to be the 
Euler-Satake characteristic of the $\Gamma$-sectors $\widetilde{Q}_{\Gamma}$ of $Q$, an orbifold generalizing the inertia orbifold and multisectors whose definition is given by the authors in [9]; see also their paper [8]. For a manifold $M$, the $\Gamma$-sectors of $M$ coincide with $M$ for each $\Gamma$ so that $\chi_{\Gamma}^{\mathrm{ES}}(M)=\chi_{\text {top }}(M)$, the usual Euler characteristic. For an orbifold $Q$, the relationship between the $\chi_{\Gamma}^{\mathrm{ES}}(Q)$ and other Euler characteristics for orbifolds is summarized below.

- When $Q$ is presented as the quotient $M / G$ with $G$ a finite group, the $\chi_{\Gamma}^{\mathrm{ES}}(Q)$ are the generalized orbifold Euler characteristics defined by Tamanoi in $[21 ; 22]$ where they are denoted $\chi_{\Gamma}^{\text {orb }}(M ; G)$. This follows from [8, Theorem 3.1].

- When $\Gamma=\mathbb{Z}^{m}$ and $Q$ is a global quotient, $\chi_{\mathbb{Z}^{m}}^{\mathrm{ES}}(Q)$ is the $m$-th orbifold Euler characteristic defined by Bryan and Fulman in [5]. By Equation (7) below and the fact that the $\mathbb{Z}$-sectors correspond to the inertia orbifold, this implies that the $m$-th Euler characteristic of Bryan and Fulman is the Euler-Satake characteristic of the $m$-th inertia orbifold of $Q$.

- The $\mathbb{Z}^{2}$-Euler-Satake characteristic $\chi_{\mathbb{Z}^{2}}^{\mathrm{ES}}(Q)$ coincides with the stringy orbifold Euler characteristic defined for global quotients by Dixon, Harvey, Vafa and Witten in [7] and for general orbifolds by Roan in [17]. Note that this Euler characteristic was shown to be the Euler characteristic of equivariant $K$-theory by Segal in [3] for global quotients and of orbifold $K$-theory by Adem and Ruan in [2] for quotients by compact Lie groups.

- When $\Gamma=\{1\}, \widetilde{Q}_{\Gamma}=Q$ so that $\chi_{\Gamma}^{\mathrm{ES}}(Q)$ is the usual Euler-Satake characteristic.

Our main results are as follows. Theorem 3.1 demonstrates that recursively constructing $\Gamma$-sectors corresponds to the direct product operation for groups. This allows us to relate the $\Gamma$-Euler-Satake characteristics to the usual (topological) Euler characteristic of the underlying space of the orbifold in question. We then prove Theorem 5.11, establishing a formula for the $\mathbb{Z}^{m}$-Euler-Satake characteristic of the wreath symmetric product of an orbifold presented as a quotient by the locally free action of a compact, connected Lie group, in particular including all closed, effective orbifolds. Theorem 6.4 gives a formula for the (shifted) Hodge numbers of wreath symmetric products of the locally free quotient by a compact, complex, connected Lie group action, including all closed, effective, complex orbifolds. These results generalize corresponding results for quotients by finite groups in Tamanoi [21], Wang [25] and Wang and Zhou [26].

In Sections 2, 3 and 4, we consider arbitrary closed orbifolds presented by an (étale) orbifold groupoid $\mathcal{G}$. In Section 2, we recall the relevant preliminary material and fix notation. In particular, in Section 2.1, we recall the definition of $\Gamma$-sectors, and in Section 2.2, we verify the multiplicativity and other basic properties of the EulerSatake characteristic. Section 3 establishes the behavior of the $\Gamma$-sectors of a general 
orbifold under product operations on both the orbifold and the group $\Gamma$. In Section 4, we define the $\Gamma$-Euler-Satake characteristics and verify the properties that indicate their connections with other Euler characteristics for orbifolds described above. In Section 5, we turn our attention to wreath symmetric products of orbifolds presented by transformation groupoids $M \rtimes G$ where $G$ is a compact, connected Lie group acting smoothly, effectively and locally freely on the smooth manifold $M$ and prove Theorem 5.11. Section 6 turns to the case of complex orbifolds given by quotients by compact, complex, connected Lie groups, proving Theorem 6.4. Throughout, $\Gamma, \Gamma_{i}$ for $i=1,2, \Lambda$ and $\Gamma^{\prime}$ will denote finitely generated discrete groups, and all manifolds, group actions and orbifolds are assumed to be smooth.

If $Q$ is a closed, effective orbifold, then $Q$ can always be presented by the smooth, locally free action of a compact, connected Lie group $G$ on a closed manifold $M$. If $Q$ is orientable, we can take $M$ to be the oriented orthonormal frame bundle and $G=\mathrm{SO}(n)$; in the nonorientable case, $M$ can be taken to be the unitary frame bundle of the complexified tangent space and $G=U(n)$; see Moerdijk and Mrčun [15, Propositions 2.22 and 2.23]. Similarly, any closed, effective, complex orbifold can be presented by the locally free action of $U(n)$ on its unitary frame bundle. For this reason, when dealing with quotient orbifolds, we always assume that the Lie group is compact and connected for simplicity, though some of our arguments extend to locally free actions of more general groups. As the $\Gamma$-sectors and Euler-Satake characteristic are Morita invariants, the results of Section 5 apply to any closed, effective orbifold, and those of Section 6 apply to any closed, effective, complex orbifold.

Acknowledgements The first author would like to thank the MSRI for its hospitality during the preparation of this manuscript. The second author was partially supported by a Rhodes College Faculty Development Endowment Grant. We would like to express our appreciation to the referee for helpful corrections and suggestions.

\section{Preliminaries}

In this section, we briefly recall the definitions we will need. For background on orbifolds, the reader is referred to Adem, Leida and Ruan [1]; see also Moerdijk and Mrčun [15], Moerdijk [14], Chen and Ruan [6] and Ruan [18]. More details on the definition of the $\Gamma$-sectors of an orbifold are presented by the authors in $[9 ; 8]$.

\subsection{Orbifolds and $\Gamma$-sectors}

An orbifold structure on a paracompact Hausdorff space $\mathbb{X}_{Q}$ is an orbifold groupoid $\mathcal{G}$, ie a proper étale Lie groupoid, and a homeomorphism $f:|\mathcal{G}| \rightarrow \mathbb{X}_{Q}$ between the 
orbit space $|\mathcal{G}|$ of $\mathcal{G}$ and $\mathbb{X}_{Q}$. We say that $(\mathcal{G}, f)$ is a presentation of the orbifold structure. Two presentations $(\mathcal{G}, f)$ and $\left(\mathcal{G}^{\prime}, f^{\prime}\right)$ are equivalent if $\mathcal{G}$ and $\mathcal{G}^{\prime}$ are Morita equivalent and the homeomorphism $g:|\mathcal{G}| \rightarrow\left|\mathcal{G}^{\prime}\right|$ induced by the Morita equivalence satisfies $f=f^{\prime} \circ g$. An orbifold $Q$ is a paracompact Hausdorff space $\mathbb{X}_{Q}$, called the underlying space of $Q$, and an equivalence class of orbifold structures on $\mathbb{X}_{Q}$. Given a presentation $(\mathcal{G}, f)$ of the orbifold $Q$, we will often identify $\mathbb{X}_{Q}$ with $|\mathcal{G}|$ and avoid explicit reference to $f$. We say that orbifolds $Q_{1}$ and $Q_{2}$ are orbifold-diffeomorphic or simply diffeomorphic if the groupoids representing them are Morita equivalent, so that in particular their underlying spaces are homeomorphic.

Let $Q$ be an orbifold. Throughout, we use the notation that $\mathcal{G}$ is an orbifold groupoid presenting $Q$ with space of objects $G_{0}$ and space of arrows $G_{1}$. When considering wreath symmetric products, we will restrict our attention to orbifolds $Q$ presented by $M \rtimes G$ where $G$ is a compact, connected Lie group acting smoothly, effectively and locally freely (ie properly with discrete stabilizers) on the smooth manifold $M$ so that $M \rtimes G$ is Morita equivalent to an orbifold groupoid. An orbifold presented by the locally free action of a Lie group on a smooth manifold is called a quotient orbifold and a global quotient orbifold if $G$ is finite. Note that $M \rtimes G$ is not étale unless $G$ is finite. For clarity, we will represent general orbifolds in terms of left groupoid actions and quotient orbifolds in terms of right group actions.

For every $x \in G_{0}$, there is an open neighborhood $V_{x} \subseteq G_{0}$ of $x$ diffeomorphic to $\mathbb{R}^{n}$ with $x$ corresponding to the origin such that the isotropy group $G_{x}$ acts linearly on $V_{x}$ and the restriction $\left.\mathcal{G}\right|_{V_{x}}$ is isomorphic to $G_{x} \ltimes V_{x}$. We let $\pi_{x}: V_{x} \rightarrow\left|G_{x} \ltimes V_{x}\right| \subseteq|\mathcal{G}|$ denote the quotient map and refer to the triple $\left\{V_{x}, G_{x}, \pi_{x}\right\}$ as a linear orbifold chart for $Q$ at $x$.

Let $\mathcal{G}$ and $\mathcal{H}$ be orbifold groupoids. By $\mathcal{G} \times \mathcal{H}$, we mean the groupoid with objects $G_{0} \times H_{0}$ and arrows $G_{1} \times H_{1}$; see Moerdijk and Mrčun [15, page 123]. As the product of proper maps is proper and the product of local diffeomorphism is a local diffeomorphism, $\mathcal{G} \times \mathcal{H}$ is an orbifold groupoid. Linear charts for this orbifold are given by products of linear charts for $|\mathcal{G}|$ and $|\mathcal{H}|$.

Given a finitely generated discrete group $\Gamma$, the space $\mathcal{S}_{\mathcal{G}}^{\Gamma}=\operatorname{Hom}(\Gamma, \mathcal{G})$ of groupoid homomorphisms from $\Gamma$ into $\mathcal{G}$ inherits the structure of a smooth $\mathcal{G}$-manifold. We let $\mathcal{G}^{\Gamma}=\mathcal{G} \ltimes \mathcal{S}_{\mathcal{G}}^{\Gamma}$ denote the corresponding orbifold groupoid and $\widetilde{Q}_{\Gamma}$ the corresponding orbifold. We refer to $\widetilde{Q}_{\Gamma}$ as the orbifold of $\Gamma$-sectors of $Q$. If $\phi_{x}: \Gamma \rightarrow G_{x}$ is an element of $\mathcal{S}_{\mathcal{G}}^{\Gamma}$, we let $\widetilde{Q}_{(\phi)}$ denote connected component of $\widetilde{Q}_{\Gamma}$ containing the orbit of $\phi_{x}$ and refer to $\widetilde{Q}_{(\phi)}$ as the $\Gamma$-sector associated to $\phi_{x}$.

Given a homomorphism $\phi_{x}: \Gamma \rightarrow G_{x}$ and a linear chart $\left\{V_{x}, G_{x}, \pi_{x}\right\}$ for $Q$ near $x$, there is a diffeomorphism $\kappa_{\phi_{x}}: V_{x}^{\left\langle\phi_{x}\right\rangle} \rightarrow \mathcal{S}_{\mathcal{G}}^{\Gamma}$ onto a neighborhood of $\phi_{x}$, where $V_{x}^{\left\langle\phi_{x}\right\rangle}$ 
denotes the subspace of $V_{x}$ fixed by the image of $\phi_{x}$. Then up to identification via $\kappa_{\phi_{x}}$, $\left\{V_{x}^{\left\langle\phi_{x}\right\rangle}, C_{G_{x}}\left(\phi_{x}\right), \pi_{x}^{\phi_{x}}\right\}$ forms a linear chart for the groupoid $\mathcal{G}^{\Gamma}=\mathcal{G} \ltimes \mathcal{S}_{\mathcal{G}}^{\Gamma}$ at $\phi_{x}$ where $C_{G_{x}}\left(\phi_{x}\right)$ is the centralizer of $\operatorname{Im} \phi_{x}$ in $G_{x}$ and $\pi_{x}^{\phi_{x}}$ is the quotient map.

In the case that $Q$ is presented by $M \rtimes G$ where $M$ is a smooth manifold and $G$ is a compact Lie group acting smoothly and locally freely, the space of $\Gamma$-sectors admits the presentation

$$
(M ; G)_{\Gamma}:=\coprod_{(\phi) \in t_{M ; G}^{\Gamma}} M^{\langle\phi\rangle} \rtimes C_{G}(\phi)
$$

where $t_{M ; G}^{\Gamma}$ denotes the set of conjugacy classes of homomorphisms $\phi \in \operatorname{Hom}(\Gamma, G)$ such that $M^{\langle\phi\rangle} \neq \varnothing$. Note that $M^{\langle\phi\rangle} \rtimes C_{G}(\phi)$ need not be a connected orbifold.

\subsection{The Euler-Satake characteristic and its properties}

Let $Q$ be a compact orbifold of dimension $n$ and let $Q_{\text {eff }}$ be the effective orbifold associated to $Q$ (see Adem, Leida and Ruan [1, Definition 2.33]). It is well known that $Q_{\text {eff }}$ can be presented by a groupoid $M \rtimes G$ where $M$ is a manifold and $G$ a compact Lie group acting smoothly and locally freely on $M$. By Illman [11, page 488] (see also Yang [27] and Verona [24]), it follows that $Q_{\text {eff }}$ admits a good finite triangulation; ie a finite triangulation for which the $G$-isotropy type is constant on the interior of each simplex. It is easy to see that a good triangulation of $Q_{\text {eff }}$ induces a triangulation of $Q$ such that the isomorphism type of the isotropy group is constant on the interior of each simplex. By refining triangulations and using stellar subdivisions if necessary, we may also assume that each of the top simplices is contained inside the image of a linear orbifold chart; see Moerdijk and Pronk [16]. Following the language for quotients, we will refer to such a triangulation of the orbifold $Q$ as good.

The following definition was originally stated in Satake [19] under the name Euler characteristic as a $V$-manifold.

Definition 2.1 (Euler-Satake characteristic) Let $Q$ be a closed orbifold and $\mathcal{T}$ a good triangulation of $Q$. The Euler-Satake characteristic of $Q$ is

$$
\chi_{\mathrm{ES}}(Q)=\sum_{\sigma \in \mathcal{T}}(-1)^{\operatorname{dim} \sigma} \frac{1}{\left|G_{\sigma}\right|},
$$

where $G_{\sigma}$ denotes the isotropy group of a point on the interior of $\sigma$. If $Q^{\prime}$ is a subset of $Q$ corresponding to a subcomplex $\mathcal{T}^{\prime}$ of $\mathcal{T}$, then we define $\chi_{\mathrm{ES}}\left(Q^{\prime}\right)$ identically, summing over those simplices contained in $\mathcal{T}^{\prime}$. If $\mathcal{G}$ is a groupoid presenting $Q$, we will use the notation $\chi_{\mathrm{ES}}(\mathcal{G})$ to denote $\chi_{\mathrm{ES}}(Q)$. 
It is clear that $\chi_{\mathrm{ES}}(Q)$ does not depend on the choice of (good) triangulation. If $Q$ is a smooth manifold, then $\chi_{\mathrm{ES}}(Q)$ is the standard Euler characteristic $\chi_{\text {top }}(Q)$ of the underlying space of $Q$. If $Q$ is a global quotient orbifold presented by $M \rtimes G$ where $G$ is a finite group, then

$$
\chi_{\mathrm{ES}}(Q)=\frac{1}{|G|} \chi_{\mathrm{top}}(M) .
$$

Similarly, it is easy to see that if $Q$ is connected and noneffective, then

$$
\chi_{\mathrm{ES}}(Q)=\frac{1}{\left|K_{p}\right|} \chi_{\mathrm{ES}}\left(Q_{\mathrm{eff}}\right)
$$

where $K_{p}$ is the isotropy group of any point $p$ that is nonsingular in $Q_{\text {eff }}$ (equivalently the normal subgroup $K_{p} \leq G_{p}$ of any isotropy group that acts trivially in a chart). In the case that $Q$ is not connected, the isomorphism class of $K_{p}$ may vary over connected components.

It is also a direct consequence of the definition that the Euler-Satake characteristic is additive; that is, if $Q_{1}$ and $Q_{2}$ are closed subsets of $Q$ such that $Q_{1} \cup Q_{2}=Q$, and the sets $Q_{1}, Q_{2}$ and $Q_{1} \cap Q_{2}$ correspond to subcomplexes of $\mathcal{T}$, then

$$
\chi_{\mathrm{ES}}\left(Q_{1} \cup Q_{2}\right)=\chi_{\mathrm{ES}}\left(Q_{1}\right)+\chi_{\mathrm{ES}}\left(Q_{2}\right)-\chi_{\mathrm{ES}}\left(Q_{1} \cap Q_{2}\right) .
$$

A covering orbifold of the orbifold $Q$ presented by the orbifold groupoid $\mathcal{G}$ is a $\mathcal{G}-$ space $E$ equipped with a connected covering projection $\rho: E \rightarrow G_{0}$; see Adem, Leida and Ruan [1, page 40]. We have the following.

Lemma 2.2 Let $\rho: \hat{Q} \rightarrow Q$ be a covering orbifold with $k$ sheets. Then

$$
\chi_{\mathrm{ES}}(\hat{Q})=k \chi_{\mathrm{ES}}(Q)
$$

See Thurston [23, Proposition 13.3.4] for the effective case, and note that the noneffective case follows from Equation (2). Note that for noneffective orbifolds, which have only singular points, the number of sheets is given by counting the preimages of a point with minimal isotropy including multiplicity.

Theorem 2.3 (Multiplicativity of the Euler-Satake characteristic) Let $Q_{1}$ and $Q_{2}$ be compact orbifolds. Then

$$
\chi_{\mathrm{ES}}\left(Q_{1} \times Q_{2}\right)=\chi_{\mathrm{ES}}\left(Q_{1}\right) \chi_{\mathrm{ES}}\left(Q_{2}\right) .
$$

Proof We first claim that Equation (4) holds when $Q_{1}$ is a compact manifold, possibly with boundary, and $Q_{2}$ is a compact orbifold. The proof of this claim follows Kawakubo [12, page 260]. Let $n$ denote the dimension of $Q_{2}$, let $\mathcal{T}_{1}$ be a finite 
triangulation of $Q_{1}$, and let $\mathcal{T}_{2}$ be a good finite triangulation of $\mathcal{T}_{2}$. For $i=1,2$, we let $\mathcal{T}_{i}^{t}$ denote the $t$-skeleton of $\mathcal{T}_{i}$. Fix $\sigma^{t} \in \mathcal{T}_{2}$, and let $\left\{V_{\sigma^{t}}, G_{\sigma^{t}}, \pi_{\sigma^{t}}\right\}$ be a linear orbifold chart for $Q_{2}$ whose image contains $\sigma^{t}$. Let $\tilde{\sigma}^{t}=\pi^{-1}\left(\sigma^{t}\right)$, and then $\pi_{\sigma^{t}}: \widetilde{\sigma}^{t} \rightarrow \sigma^{t}$ is an orbifold cover. It follows that $Q_{1} \times \sigma^{t}$ is presented by $\left(Q_{1} \times \widetilde{\sigma}^{t}\right) \rtimes G_{\sigma^{t}}$ with $G_{\sigma^{t}}$ acting trivially on the first factor. Hence, by Equation (1), the multiplicative property of $\chi_{\mathrm{top}}$, and the multiplicative property of $\chi_{\mathrm{ES}}$ on orbifold covers, we have

$$
\begin{aligned}
\chi_{\mathrm{ES}}\left(Q_{1} \times \sigma^{t}\right) & =\chi_{\mathrm{ES}}\left(\left(Q_{1} \times \tilde{\sigma}^{t}\right) \rtimes G_{\sigma^{t}}\right) \\
& =\chi_{\mathrm{top}}\left(Q_{1} \times \tilde{\sigma}^{t}\right) /\left|G_{\sigma^{t}}\right| \\
& =\chi_{\mathrm{top}}\left(Q_{1}\right) \chi_{\mathrm{top}}\left(\widetilde{\sigma}^{t}\right) /\left|G_{\sigma^{t}}\right| \\
& =\chi_{\mathrm{ES}}\left(Q_{1}\right) \chi_{\mathrm{ES}}\left(\sigma^{t}\right) .
\end{aligned}
$$

Applying this to each $\sigma \in \mathcal{T}_{2}$ and using Equation (3), we have

$$
\begin{aligned}
\chi_{\mathrm{ES}}\left(Q_{1} \times Q_{2}\right)= & \sum_{\sigma^{n} \in \mathcal{T}_{2}^{n}} \chi_{\mathrm{ES}}\left(Q_{1} \times \sigma^{n}\right)-\sum_{\sigma^{n-1} \in \mathcal{T}_{2}^{n-1}} \chi_{\mathrm{ES}}\left(Q_{1} \times \sigma^{n-1}\right) \\
& +\sum_{\sigma^{n-2} \in \mathcal{T}_{2}^{n-2}} \chi_{\mathrm{ES}}\left(Q_{1} \times \sigma^{n-2}\right)+\cdots+(-1)^{n} \sum_{\sigma^{0} \in \mathcal{T}_{2}^{0}} \chi_{\mathrm{ES}}\left(Q_{1} \times \sigma^{0}\right) \\
= & \sum_{t=0}^{n}(-1)^{n-t} \sum_{\sigma^{t} \in \mathcal{T}_{2}^{t}} \chi_{\mathrm{ES}}\left(Q_{1} \times \sigma^{t}\right) \\
= & \sum_{t=0}^{n}(-1)^{n-t} \sum_{\sigma^{t} \in \mathcal{T}_{2}^{t}} \chi_{\mathrm{ES}}\left(Q_{1}\right) \chi_{\mathrm{ES}}\left(\sigma^{t}\right) \\
= & \chi_{\mathrm{ES}}\left(Q_{1}\right) \sum_{t=0}^{n}(-1)^{n-t} \sum_{\sigma^{t} \in \mathcal{T}_{2}^{t}} \chi_{\mathrm{ES}}\left(\sigma^{t}\right) \\
= & \chi_{\mathrm{ES}}\left(Q_{1}\right) \chi_{\mathrm{ES}}\left(Q_{2}\right),
\end{aligned}
$$

proving the claim.

In general, let $\mathcal{T}_{1}$ and $\mathcal{T}_{2}$ be good finite triangulations of $Q_{1}$ and $Q_{2}$, respectively. By applying the claim to each $\sigma^{t} \in \mathcal{T}_{2}$ as well as multiplicativity on orbifold covers, we have

$$
\begin{aligned}
\chi_{\mathrm{ES}}\left(Q_{1} \times \sigma^{t}\right) & =\chi_{\mathrm{ES}}\left(\left(Q_{1} \times \tilde{\sigma}^{t}\right) \rtimes G_{\widetilde{\sigma}^{t}}\right) \\
& =\chi_{\mathrm{ES}}\left(Q_{1} \times \widetilde{\sigma}^{t}\right) / \mid G_{\widetilde{\sigma}^{t} \mid} \\
& =\chi_{\mathrm{ES}}\left(Q_{1}\right) \chi_{\mathrm{ES}}\left(\widetilde{\sigma}^{t}\right) / \mid G_{\widetilde{\sigma}^{t} \mid} \\
& =\chi_{\mathrm{ES}}\left(Q_{1}\right) \chi_{\mathrm{ES}}\left(\sigma^{t}\right) .
\end{aligned}
$$

Summing up over all of the simplices in $\mathcal{T}_{2}$ and applying Equation (3) as in Equation (5), Equation (4) follows. 


\section{Operations on $\Gamma$-sectors}

In this section, we examine operations on groups and orbifolds and the corresponding operations on the $\Gamma$-sectors. Note that in [8, Lemma 2.6], the authors observed that if $\mathcal{G}$ is a groupoid and $e_{0}: M_{1} \rightarrow M_{2}$ is a $\mathcal{G}$-equivariant map of $\mathcal{G}$-spaces, then there is a groupoid homomorphism $e: \mathcal{G} \ltimes M_{1} \rightarrow \mathcal{G} \ltimes M_{2}$ with $e_{0}$ the map on objects and

$$
\begin{aligned}
e_{1}:\left(\mathcal{G} \ltimes M_{1}\right)_{1} & \longrightarrow\left(\mathcal{G} \ltimes M_{2}\right)_{1} \\
(g, z) & \longmapsto\left(g, e_{0}(z)\right)
\end{aligned}
$$

the map on arrows. In particular, if $e_{0}$ is a bijection then $e$ is an isomorphism, if $e_{0}$ is smooth then $e$ is a homomorphism of Lie groupoids and if $e_{0}$ is a diffeomorphism then $e$ is an isomorphism of Lie groupoids. We make frequent use of these facts below.

\subsection{The $\Gamma_{1} \times \Gamma_{2}$-sectors}

Let $\Gamma_{1}$ and $\Gamma_{2}$ be finitely generated discrete groups. Since $\widetilde{Q}_{\Gamma_{1}}$ is an orbifold with orbifold structure given by the groupoid $\mathcal{G}^{\Gamma}=\mathcal{G} \ltimes \mathcal{S}_{\mathcal{G}}^{\Gamma_{1}}$, it makes sense to form the $\Gamma_{2}$-sectors of this orbifold as the translation groupoid $\left(\mathcal{G} \ltimes \mathcal{S}_{\mathcal{G}}^{\Gamma_{1}}\right) \ltimes \mathcal{S}_{\mathcal{G} \ltimes \mathcal{S}_{\mathcal{G}}^{\Gamma_{1}}}^{\Gamma_{2}}$. We denote the resulting orbifold by $\left(\widetilde{Q}_{\Gamma_{1}}\right)_{\Gamma_{2}}$. By the authors' results [8, Theorems 3.1 and 3.5], the following is a generalization of Tamanoi [22, Proposition 2-1 (2)].

Theorem 3.1 Let $\mathcal{G}$ be a groupoid and $\Gamma_{1}$ and $\Gamma_{2}$ be groups. There is a groupoid isomorphism from

$$
\left(\mathcal{G} \ltimes \mathcal{S}_{\mathcal{G}}^{\Gamma_{1}}\right) \ltimes \mathcal{S}_{\mathcal{G} \ltimes \mathcal{S}_{\mathcal{G}}^{\Gamma_{1}}}^{\Gamma_{1}} \quad \text { to } \quad \mathcal{G} \ltimes \mathcal{S}_{\mathcal{G}}^{\Gamma_{1} \times \Gamma_{2}} .
$$

In particular, if $\mathcal{G}$ is an orbifold groupoid, then the orbifolds $\widetilde{\left(\widetilde{Q}_{\Gamma_{1}}\right)_{\Gamma_{2}}}$ and $\widetilde{Q}_{\Gamma_{1} \times \Gamma_{2}}$ are diffeomorphic.

Proof First, we demonstrate a bijection between the spaces of objects. An element of $\mathcal{S}_{\mathcal{G}}^{\Gamma_{1} \times \Gamma_{2}}$ is a homomorphism $\Phi_{x}: \Gamma_{1} \times \Gamma_{2} \rightarrow G_{x}$ where $x \in G_{0}$. An element of $\mathcal{S}_{\mathcal{G} \ltimes \mathcal{S}_{\mathcal{G}}^{\Gamma_{1}}}^{\Gamma_{2}}$ is a homomorphism $\psi_{\phi_{x}}$ from $\Gamma_{2}$ into the isotropy group $C_{G_{x}}\left(\phi_{x}\right)$ of the point $\phi_{x} \in \mathcal{S}_{\mathcal{G}}^{\Gamma_{1}}$ with respect to the groupoid $\mathcal{G} \ltimes \mathcal{S}_{\mathcal{G}}^{\Gamma_{1}}$.

Fix $x \in G_{0}$; we claim there is a bijection between homomorphisms $\Phi_{x}: \Gamma_{1} \times \Gamma_{2} \rightarrow G_{x}$ and pairs of homomorphisms $\phi_{x}: \Gamma_{1} \rightarrow G_{x}$ and $\psi_{\phi_{x}}: \Gamma_{2} \rightarrow C_{G_{x}}\left(\phi_{x}\right)$. Let $\phi_{x}: \Gamma_{1} \rightarrow G_{x}$ and $\psi_{\phi_{x}}: \Gamma_{2} \rightarrow C_{G_{x}}\left(\phi_{x}\right)$. We define $\phi_{x} \cdot \psi_{\phi_{x}}$ to be the pointwise product, ie

$$
\begin{aligned}
\phi_{x} \cdot \psi_{\phi_{x}}: & \Gamma_{1} \times \Gamma_{2} \\
\left(\gamma_{1}, \gamma_{2}\right) & \longmapsto G_{x} \\
& \longmapsto \phi_{x}\left(\gamma_{1}\right) \psi_{\phi_{x}}\left(\gamma_{2}\right) .
\end{aligned}
$$


As $\psi_{\phi_{x}}\left(\gamma_{2}\right) \in C_{G_{x}}\left(\phi_{x}\right)$ for each $\gamma_{2} \in \Gamma_{2}$, it is clear that $\phi_{x} \cdot \psi_{\phi_{x}}$ is a homomorphism. Given a homomorphism $\Phi_{x}: \Gamma_{1} \times \Gamma_{2} \rightarrow G_{x}$, if we set $\phi_{x}\left(\gamma_{1}\right)=\Phi_{x}\left(\gamma_{1}, 1\right)$ for each $\gamma_{1} \in \Gamma_{1}$ and $\psi_{\phi_{x}}\left(\gamma_{2}\right)=\Phi_{x}\left(1, \gamma_{2}\right)$ for each $\gamma_{2} \in \Gamma_{2}$, then $\Phi_{x}=\phi_{x} \cdot \psi_{\phi_{x}}$, with $\psi_{\phi_{x}}\left(\gamma_{2}\right) \in C_{G_{x}}\left(\phi_{x}\right)$ for each $\gamma_{2} \in \Gamma_{2}$. It follows that the map

$$
e_{0}: \mathcal{S}_{\mathcal{G} \ltimes \mathcal{S}_{\mathcal{G}}^{\Gamma_{1}}}^{\Gamma_{1}} \longrightarrow \mathcal{S}_{\mathcal{G}}^{\Gamma_{1} \times \Gamma_{2}}, \quad \psi_{\phi_{x}} \longmapsto \phi_{x} \cdot \psi_{\phi_{x}}
$$

is bijective.

Now we represent $\left(\mathcal{G} \ltimes \mathcal{S}_{\mathcal{G}}^{\Gamma_{1}}\right) \ltimes \mathcal{S}_{\mathcal{G} \ltimes \mathcal{S}_{\mathcal{G}}^{\Gamma_{1}}}$ as a $\mathcal{G}$-space. An arrow in $\mathcal{G} \ltimes \mathcal{S}_{\mathcal{G}}^{\Gamma_{1}}$ is given by a pair $\left(h, \phi_{x}\right)$ with $\phi_{x} \in \mathcal{S}_{\mathcal{G}}^{\Gamma_{1}}$ and an $h \in \mathcal{G}$ such that $s(h)=x$. Then for each $\psi_{\phi_{x}} \in \mathcal{S}_{\mathcal{G} \ltimes \mathcal{S}_{\mathcal{G}}^{\Gamma_{1}}}^{\Gamma_{2}}$ and $\gamma_{2} \in \Gamma_{2}$, the action is given by

$$
\left[\left(h, \phi_{x}\right) \cdot \psi_{\phi_{x}}\right]\left(\gamma_{2}\right)=h \psi_{\phi_{x}}\left(\gamma_{2}\right) h^{-1}
$$

where $h \psi_{\phi_{x}}\left(\gamma_{2}\right) h^{-1}$ is a homomorphism from $\Gamma_{2}$ to $C_{G_{t(h)}}\left(h \phi_{x} h^{-1}\right)$. So, if we let $\alpha: \mathcal{S}_{\mathcal{G} \ltimes \mathcal{S}_{\mathcal{G}} \Gamma_{1}}^{\Gamma_{1}} \rightarrow G_{0}$ be defined by $\alpha\left(\psi_{\phi_{x}}\right)=x$, then $\alpha$ is the anchor map of a $\mathcal{G}$-action

$$
\left[h \cdot \psi_{\phi_{x}}\right]\left(\gamma_{2}\right)=h \psi_{\phi_{x}}\left(\gamma_{2}\right) h^{-1}
$$

defined whenever $\alpha\left(\psi_{\phi_{x}}\right)=s(h)=x$. The requirements of a groupoid action are clearly satisfied, so that $\left(\mathcal{G} \ltimes \mathcal{S}_{\mathcal{G}}^{\Gamma_{1}}\right) \ltimes \mathcal{S}_{\mathcal{G} \ltimes \mathcal{S}_{\mathcal{G}}^{\Gamma_{1}}}$ is isomorphic to $\mathcal{G} \ltimes \mathcal{S}_{\mathcal{G} \ltimes \mathcal{S}_{\mathcal{G}}^{\Gamma_{1}}}^{\Gamma_{1}}$.

With this, note that for each $h \in G_{1}$ with $s(h)=x$, each $\psi_{\phi_{x}} \in \mathcal{S}_{\mathcal{G}_{2} \ltimes \mathcal{S}_{\mathcal{G}}}^{\Gamma_{1}}$, each $\gamma_{1} \in \Gamma_{1}$ and $\gamma_{2} \in \Gamma_{2}$, one obtains via a straightforward calculation that

$$
e_{0}\left[h \cdot \psi_{\phi_{x}}\right]\left(\gamma_{1}, \gamma_{2}\right)=\left[h \cdot e_{0}\left(\psi_{\phi_{x}}\right)\right]\left(\gamma_{1}, \gamma_{2}\right)
$$

It follows that $e_{0}$ is $\mathcal{G}$-equivariant, and hence by [8, Lemma 2.6] is the map on objects of a groupoid isomorphism. The map on arrows is given by

$$
e_{1}: \mathcal{G} \ltimes \mathcal{S}_{\mathcal{G} \ltimes \mathcal{S}_{\mathcal{G}}^{\Gamma_{1}}}^{\Gamma_{1}} \longrightarrow \mathcal{G} \ltimes \mathcal{S}_{\mathcal{G}}^{\Gamma_{1} \times \Gamma_{2}}, \quad\left(h, \psi_{\phi_{x}}\right) \longmapsto\left(h, \phi_{x} \cdot \psi_{\phi_{x}}\right) .
$$

When $\mathcal{G}$ is an orbifold groupoid, a straightforward argument using local charts shows that $e_{0}$ is smooth, which again by $[8$, Lemma 2.6] completes the proof.

\subsection{The $\Gamma$-sectors of product orbifolds}

In this subsection, we prove the following.

Proposition 3.2 Suppose $\mathcal{G}$ and $\mathcal{H}$ are groupoids and $\Gamma$ is a group. Then $(\mathcal{G} \times \mathcal{H})^{\Gamma}$ and $\mathcal{G}^{\Gamma} \times \mathcal{H}^{\Gamma}$ are isomorphic. If $\mathcal{G}$ and $\mathcal{H}$ are orbifold groupoids, then the isomorphism is of orbifold groupoids. 
Note that this in particular implies that if $Q_{1}$ is presented by $\mathcal{G}$ and $Q_{2}$ is presented by $\mathcal{H}$, then $\widetilde{\left(Q_{1} \times Q_{2}\right)} \Gamma$ and $\widetilde{\left(Q_{1}\right)_{\Gamma}} \times \widetilde{\left(Q_{2}\right)_{\Gamma}}$ are diffeomorphic orbifolds, generalizing Tamanoi [22, Proposition 2-1 (1)] to the case of general orbifold groupoids.

Proof Recall that $(\mathcal{G} \times \mathcal{H})^{\Gamma}=(\mathcal{G} \times \mathcal{H}) \ltimes\left(\mathcal{S}_{\mathcal{G}}^{\Gamma} \times \mathcal{S}_{\mathcal{H}}^{\Gamma}\right)$ and $\mathcal{G}^{\Gamma} \times \mathcal{H}^{\Gamma}=\left(\mathcal{G} \ltimes \mathcal{S}_{\mathcal{G}}^{\Gamma}\right) \times\left(\mathcal{H} \ltimes \mathcal{S}_{\mathcal{H}}^{\Gamma}\right)$. Note that an element of $\mathcal{S}_{\mathcal{G}}^{\Gamma} \times \mathcal{S}_{\mathcal{H}}^{\Gamma}$ is of the form $\left(\phi_{x}, \psi_{y}\right)$ for $x \in G_{0}, y \in H_{0}$, $\phi_{x} \in \operatorname{Hom}\left(\Gamma, G_{x}\right)$ and $\psi_{y} \in \operatorname{Hom}\left(\Gamma, H_{y}\right)$. Define the map

$$
e_{0}: \mathcal{S}_{\mathcal{G}}^{\Gamma} \times \mathcal{S}_{\mathcal{H}}^{\Gamma} \longrightarrow \mathcal{S}_{\mathcal{G} \times \mathcal{H}}^{\Gamma}, \quad\left(\phi_{x}, \psi_{y}\right) \longmapsto \phi_{x} \times \psi_{y}
$$

where $\phi_{x} \times \psi_{y} \in \operatorname{Hom}\left(\Gamma, G_{x} \times H_{y}\right)$ is defined by

$$
\left(\phi_{x} \times \psi_{y}\right)(\gamma)=\left(\phi_{x}(\gamma), \psi_{y}(\gamma)\right) \in G_{x} \times H_{y} .
$$

That $e_{0}$ is bijective is obvious.

Now, note that the componentwise $\mathcal{G}$ - and $\mathcal{H}$-actions defining $\mathcal{G}^{\Gamma} \times \mathcal{H}^{\Gamma}$ correspond to commuting $\mathcal{G}$ - and $\mathcal{H}$-actions on $\mathcal{S}_{\mathcal{G}}^{\Gamma} \times \mathcal{S}_{\mathcal{H}}^{\Gamma}$. Hence, the $\mathcal{G} \times \mathcal{H}$-actions on $\mathcal{S}_{\mathcal{G}}^{\Gamma} \times \mathcal{S}_{\mathcal{H}}^{\Gamma}$ and $\mathcal{S}_{\mathcal{G} \times \mathcal{H}}^{\Gamma}$ coincide via $e_{0}$. Then by [8, Lemma 2.6], $e_{0}$ induces a groupoid isomorphism. When $\mathcal{G}$ and $\mathcal{H}$ are orbifold groupoids, it is easy to see by restricting to local charts that $e_{0}$ is a diffeomorphism. It follows that $e$ is an isomorphism of orbifold groupoids.

In [9], the authors define an equivalence relation $\approx$ on the $\mathcal{S}_{\mathcal{G}}^{\Gamma}$, and it was shown that $\phi_{x} \approx \psi_{y}$ if and only if the $\mathcal{G}$-orbits of $\phi_{x}$ and $\psi_{y}$ are on the same connected component of $\left|\mathcal{G}^{\Gamma}\right|$. The $\approx$-class of $\phi_{x}$ is denoted $(\phi) \approx$ or simply $(\phi)$ if no confusion is introduced. Then $T_{Q}^{\Gamma}$ was defined to be the set of $\approx$-classes of $\mathcal{S}_{\mathcal{G}}^{\Gamma}$.

As a consequence of Proposition 3.2, the connected components of $\overline{\left(Q_{1} \times Q_{2}\right)} \Gamma$ clearly correspond to products of connected components of $\overline{\left(Q_{1}\right)_{\Gamma}}$ and connected components of $\widetilde{\left(Q_{2}\right)} \Gamma$. Hence, there is a bijection between $T_{Q_{1}}^{\Gamma} \times T_{Q_{2}}^{\Gamma}$ and $T_{Q_{1} \times Q_{2}}^{\Gamma}$ as sets. This bijection is evidently given by

$$
\left((\phi)_{\approx, \mathcal{G}^{\Gamma}},(\psi)_{\approx, \mathcal{H}} \Gamma\right) \mapsto(\phi \times \psi)_{\approx,(\mathcal{G} \times \mathcal{H})^{\Gamma}}
$$

\subsection{Maps on $\Gamma$-sectors induced by group homomorphisms}

Lemma 3.3 Let $\mathcal{G}$ be a groupoid, let $\Lambda$ and $\Gamma$ be groups, and let $\Phi: \Lambda \rightarrow \Gamma$ be a group homomorphism. Then $\Phi$ induces a groupoid homomorphism

$$
e_{\Phi}: \mathcal{G}^{\Gamma} \longrightarrow \mathcal{G}^{\Lambda}
$$

If $\mathcal{G}$ is an orbifold groupoid, then $e_{\Phi}$ is a homomorphism of orbifold groupoids, and the map $e_{\Phi_{0}}$ on objects is an immersion. 
Proof The map induced by $\Phi$ on objects is given by

$$
e_{\Phi_{0}}: \mathcal{S}_{\mathcal{G}}^{\Gamma} \longrightarrow \mathcal{S}_{\mathcal{G}}^{\Lambda}, \quad \phi_{x} \longmapsto \phi_{x} \circ \Phi
$$

To check that $e_{\Phi_{0}}$ is $\mathcal{G}$-equivariant is a straightforward verification similar to those given above. In the case of an orbifold groupoid, it is easy to see that with respect to a local chart, the map $e_{\Phi_{0}}$ restricts to the embedding of $V_{x}^{\left\langle\phi_{x}\right\rangle}$ into $V_{x}^{\left\langle e_{\Phi_{0}}\left(\phi_{x}\right)\right\rangle}$. Again by $[8$, Lemma 2.6], the result follows.

We restrict our attention to the case of an orbifold groupoid $\mathcal{G}$. Using the identification of the multisectors with the $\Gamma$-sectors corresponding to free groups observed by the authors in [8, Proposition 3.7], the maps $e_{i_{1}, \ldots, i_{l}}$ defined in Adem, Leida and Ruan [1, page 80] are special cases of the construction above. In particular, let the free group $\mathbb{F}_{k}$ have generators $\gamma_{1}, \ldots, \gamma_{k}$, and pick $\left\{i_{1}, \ldots i_{l}\right\} \subseteq\{1,2, \ldots, k\}$. Define the homomorphism $\Phi_{i_{1}, \ldots, i_{l}}: \mathbb{F}_{l} \rightarrow \mathbb{F}_{k}$ by $\gamma_{1} \mapsto \gamma_{i_{1}}$, and then $e_{i_{1}, \ldots, i_{l}}=e_{\Phi_{i_{1}, \ldots, i_{l}}}$.

The homomorphism $e_{\Phi}$ induces a well-defined map $\left|e_{\Phi}\right|:\left|\mathcal{G}^{\Gamma}\right| \rightarrow\left|\mathcal{G}^{\Lambda}\right|$ on orbit spaces. Letting (1) denote the $\approx$-class of the trivial homomorphism mapping $\Gamma$ to a unit in $\mathcal{G}, \widetilde{Q}_{(1)}$ is obviously diffeomorphic to $Q$. Recall that the map $\pi: \widetilde{Q}_{\Gamma} \rightarrow Q$ was defined in [9] by $\pi\left(\mathcal{G} \phi_{x}\right)=\mathcal{G} x$. Letting $\iota:\{1\} \rightarrow \Gamma$, we see that $\pi=\left|e_{\iota}\right|$ up to the identification of $\widetilde{Q}_{(1)}$ with $Q$.

In [9] following Lemma 3.6, it was stated that the map $\pi$ is not an embedding of the $\Gamma$-sectors of $Q$ into $Q$. By this, it was meant that the restriction of $\pi$ to a $\Gamma$-sector is not generally injective unless the local groups of $Q$ are abelian. Using the definition of embeddings of orbifold groupoids in Adem, Leida and Ruan [1, Definition 2.3], the restriction of this map to a $\Gamma$-sector is in fact an embedding.

Lemma 3.4 Let $\mathcal{G}$ be an orbifold groupoid and $\Gamma$ a finitely generated discrete group. Let $(\phi) \in T_{Q}^{\Gamma}$ and $\iota:\{1\} \rightarrow \Gamma$, and then the restriction of the map $e_{\iota}$ to $(\phi)$ is an embedding of orbifold groupoids.

Proof Let $x \in G_{0}$. Then $e_{l}^{-1}(x) \cap(\phi)$ is precisely the $G_{x}$-conjugacy class of $\phi_{x}$. The number of such conjugacy classes is given by the index of $C_{G_{x}}\left(\phi_{x}\right)$ in $G_{x}$, and so for each orbifold chart $\left\{V_{x}, G_{x}, \pi_{x}\right\}$ for $Q$ at $x$, we have that $e_{\iota}^{-1}\left(V_{x}\right) \cap(\phi)$ is given by

$$
\coprod_{\psi_{x} \in\left(\phi_{x}\right)_{G_{x}}} V_{x}^{\left\langle\psi_{x}\right\rangle}=G_{x} / C_{G_{x}}\left(\phi_{x}\right) \times V_{x}^{\left\langle\psi_{x}\right\rangle},
$$

where $\left(\phi_{x}\right)_{G_{x}}$ denotes the $G_{x}$-conjugacy class of $\phi_{x}$. Moreover, $\mathcal{G}_{e_{\iota}^{-1}\left(V_{x}\right)}^{\Gamma}$ is given by

$$
\coprod_{\psi_{x} \in\left(\phi_{x}\right)_{G_{x}}} C_{G_{x}}\left(\phi_{x}\right) \ltimes V_{x}^{\left\langle\psi_{x}\right\rangle}=G_{x} \ltimes\left(G_{x} / C_{G_{x}}\left(\phi_{x}\right) \times V_{x}^{\left\langle\psi_{x}\right\rangle}\right) .
$$


As $\left|e_{\iota}\right|=\pi$ is clearly proper, it follows that the restriction of $e_{\iota}$ to each $\Gamma$-sector is an embedding.

Hence, each $\Gamma$-sector of $Q$ is a suborbifold of $Q$. We also have the following.

Lemma 3.5 Let $\mathcal{G}$ be an orbifold groupoid, let $\Lambda$ and $\Gamma$ be finitely generated discrete groups and let $\Phi: \Lambda \rightarrow \Gamma$ be a group homomorphism. If $\Phi$ maps $\Lambda$ into $\Gamma$ as a direct factor, then $e_{\Phi}$ is a finite union of embeddings of orbifold groupoids, and the induced map $\left|e_{\Phi}\right|$ on orbit spaces is surjective. In particular, $e_{\Phi}$ is an embedding of each $\Gamma$-sector into $\widetilde{Q}_{\Lambda}$. If $\Phi$ is an isomorphism, then $e_{\Phi}$ is an isomorphism of orbifold groupoids.

By virtue of [8, Proposition 3.7], this result generalizes Adem, Leida and Ruan [1, Proposition 4.1].

Proof Suppose $\Gamma=\Lambda \times \Gamma^{\prime}$ for some group $\Gamma^{\prime}$ and $\Phi: \lambda \mapsto(\lambda, 1)$. For each $\phi_{x} \in \mathcal{S}_{\mathcal{G}}^{\Lambda}$, we have $e_{\Phi_{0}}\left(\phi_{x} \times 1\right)=\phi_{x}$. It follows that $e_{\Phi_{0}}$ is surjective on objects and hence on orbit spaces.

The map $e^{-1}: \mathcal{G}^{\Gamma} \rightarrow\left(\mathcal{G}^{\Lambda}\right)^{\Gamma^{\prime}}$ given in Theorem 3.1 is an isomorphism, and hence $\mathcal{G}^{\Gamma}$ corresponds to the $\Gamma^{\prime}$-sectors of $\mathcal{G}^{\Lambda}$. Moreover, the map $\pi:\left(\mathcal{G}^{\Lambda}\right)^{\Gamma^{\prime}} \rightarrow \mathcal{G}^{\Lambda}$ clearly satisfies $\pi \circ e^{-1}=e_{\Phi}$. By Lemma 3.4, then, the restriction of $e_{\Phi}$ to each $\Gamma$-sector is an embedding.

If $e_{\Phi}$ is an isomorphism, then clearly $e_{\Phi}$ and $e_{\Phi^{-1}}$ are inverse groupoid homomorphisms.

Lemma 3.6 Let $\mathcal{G}$ be an orbifold groupoid, let $\Lambda$ and $\Gamma$ be finitely generated discrete groups and let $\Phi: \Lambda \rightarrow \Gamma$ be a group homomorphism. If $\Phi$ is surjective, then $e_{\Phi}$ is an embedding of $\mathcal{G}^{\Gamma}$ into $\mathcal{G}^{\Lambda}$ whose image consists of entire connected components.

Proof Suppose $\Phi$ is surjective. Then for each $\phi_{x} \in \mathcal{S}_{\mathcal{G}}^{\Gamma}, \operatorname{Im} \phi_{x}=\operatorname{Im} e_{\Phi_{0}}\left(\phi_{x}\right)$ as subgroups of $G_{x}$. Therefore, given a linear chart $\left\{V_{x}, G_{x}, \pi_{x}\right\}$ for $Q$ at $x$, $V_{x}^{\left\langle\phi_{x}\right\rangle}=V_{x}^{\left\langle e_{\Phi_{0}}\left(\phi_{x}\right)\right\rangle}$, and $C_{G_{x}}\left(\phi_{x}\right)=C_{G_{x}}\left(e_{\Phi_{0}}\left(\phi_{x}\right)\right)$. Moreover, if $\phi_{x}, \phi_{y}^{\prime} \in \mathcal{S}_{\mathcal{G}}^{\Gamma}$ satisfy $e_{\Phi_{0}}\left(\phi_{x}\right)=e_{\Phi_{0}}\left(\phi_{y}^{\prime}\right)$, then for each $\lambda \in \Lambda$, we have $\phi_{x}(\Phi(\lambda))=\phi_{y}^{\prime}(\Phi(\lambda))$. It clearly follows that $x=y$, and as $\Phi$ is surjective, $\phi_{x}=\phi_{y}^{\prime}$. Therefore, $e_{\Phi_{0}}$ is injective.

In local charts, the maps $e_{\Phi_{0}}$ and $e_{\Phi_{1}}$ are simply the identity maps. Hence $e_{\Phi}$ restricts in each chart to an isomorphism of orbifold groupoids. As $e_{\Phi_{0}}$ is an injective 
immersion, the induced map $\left|e_{\Phi}\right|$ on orbit spaces is clearly proper. It follows that $e_{\Phi}$ is an embedding of orbifold groupoids.

To show that the image of $\left|e_{\Phi}\right|$ consists of entire connected components, suppose $\phi_{x} \in \mathcal{S}_{\mathcal{G}}^{\Gamma}$ and $\psi_{y} \in \mathcal{S}_{\mathcal{G}}^{\Lambda}$ such that $\mathcal{G}\left(\phi_{x} \circ \Phi\right)$ and $\mathcal{G} \psi_{y}$ are elements of the same connected component of $\widetilde{Q}_{\Lambda}$. By [9, page 9], it follows that $\phi_{x} \circ \Phi \approx \psi_{y}$ as elements of $\mathcal{S}_{\mathcal{G}}^{\Lambda}$, so $\phi_{x} \circ \Phi$ and $\psi_{y}$ are connected by a sequence of local equivalences in $\mathcal{S}_{\mathcal{G}}^{\Lambda}$. Whenever

$$
\psi y \stackrel{\text { loc }}{\curvearrowright} \psi y^{\prime}
$$

there is a $g \in G_{1}$ such that $g\left[\xi_{x}^{y} \circ \psi_{y^{\prime}}(\lambda)\right] g^{-1}=\psi_{y}(\lambda)$ for each $\lambda \in \Lambda$. Therefore, $\operatorname{Im} \psi_{y}$ and $\operatorname{Im} \psi_{y^{\prime}}$ are isomorphic subgroups of $G_{y}$ and $G_{y^{\prime}}$, respectively. Hence, there exists a $\phi_{y}: \Gamma \rightarrow G_{y}$ such that $\phi_{y} \circ \Phi=\psi_{y}$ if and only if there exists a $\phi_{y^{\prime}}: \Gamma \rightarrow G_{y^{\prime}}$ such that $\phi_{y^{\prime}} \circ \Phi=\psi_{y^{\prime}}$. As this is true for each of the local equivalences connecting $\phi_{x} \circ \Phi$ to $\psi_{y}$ (regardless of their direction), it follows that there is a $\phi_{y}$ such that $\phi_{y} \circ \Phi=\psi_{y}$, and so $\psi_{y}$ is in the image of $e_{\Phi_{0}}$. It follows that $\left|e_{\Phi}\right|$ maps connected components to connected components.

It follows that when $\Phi$ is surjective, $e_{\Phi}$ embeds $\widetilde{Q}_{\Gamma}$ into $\widetilde{Q}_{\Lambda}$ as a suborbifold consisting of entire $\Lambda$-sectors of $Q$. In particular, each $\Gamma$-sector of $Q$ is diffeomorphic to a $\Lambda$-sector via $\left|e_{\Phi}\right|$.

\section{The $\Gamma$-Euler-Satake characteristics}

We state the following.

Definition 4.1 ( $\Gamma$-Euler-Satake characteristic) Let $Q$ be closed and $\Gamma$ a group. Let

$$
\chi_{\Gamma}^{\mathrm{ES}}(Q)=\sum_{(\phi) \in T_{Q}^{\Gamma}} \chi_{\mathrm{ES}}\left(\widetilde{Q}_{(\phi)}\right)=\chi_{\mathrm{ES}}\left(\widetilde{Q}_{\Gamma}\right)
$$

denote the $\Gamma$-Euler-Satake characteristic, the Euler-Satake characteristic of the space of $\Gamma$-sectors of $Q$ and

$$
\chi_{\Gamma}^{\mathrm{top}}(Q)=\sum_{(\phi) \in T_{Q}^{\Gamma}} \chi_{\mathrm{top}}\left(\widetilde{Q}_{(\phi)}\right)=\chi_{\mathrm{top}}\left(\widetilde{Q}_{\Gamma}\right)
$$

the $\Gamma$-Euler characteristic, the usual Euler characteristic of the (underlying space of the) space of $\Gamma$-sectors of $Q$. 
Note that both sums are finite when $\Gamma$ is finitely generated; this was verified by the authors in [9, Lemma 2.9]. Applying the results of the authors in [8], these definitions extend those given by Tamanoi in $[21 ; 22]$ for global quotients. In the case that $Q$ is oriented, $\chi_{\Gamma}^{\mathrm{ES}}(Q)$ is the evaluation of the $\Gamma$-Euler-Satake class of $Q$ defined in [9, Section 2.3].

We have the following two corollaries, the first a direct consequence of Theorem 3.1, and the second following from Proposition 3.2 and Theorem 2.3.

Corollary 4.2 Let $Q$ be a closed orbifold and $\Gamma_{1}$ and $\Gamma_{2}$ be finitely generated discrete groups. Then

$$
\chi_{\Gamma_{1} \times \Gamma_{2}}^{\mathrm{ES}}(Q)=\chi_{\Gamma_{1}}^{\mathrm{ES}}\left(\widetilde{Q}_{\Gamma_{2}}\right) .
$$

Corollary 4.3 Let $Q_{1}$ and $Q_{2}$ be closed orbifolds and $\Gamma$ be a finitely generated discrete group. Then

$$
\chi_{\Gamma}^{\mathrm{ES}}\left(Q_{1} \times Q_{2}\right)=\chi_{\Gamma}^{\mathrm{ES}}\left(Q_{1}\right) \chi_{\Gamma}^{\mathrm{ES}}\left(Q_{2}\right)
$$

Applying these results to the case of quotient orbifolds, we obtain the following generalization of Tamanoi [21, Proposition 2-1].

Corollary 4.4 Let $G$ be a compact Lie group, let $M$ be a smooth, compact manifold on which $G$ acts smoothly and locally freely, and let $\Gamma_{1}$ and $\Gamma_{2}$ be finitely generated discrete groups. Then

$$
\chi_{\Gamma_{1} \times \Gamma_{2}}^{\mathrm{ES}}(M \rtimes G)=\sum_{(\phi) \in \operatorname{Hom}\left(\Gamma_{1}, G\right) / G} \chi_{\Gamma_{2}}^{\mathrm{ES}}\left(M^{\langle\phi\rangle} \rtimes C_{G}(\phi)\right),
$$

and

$$
\chi_{\Gamma_{1} \times \Gamma_{2}}^{\text {top }}(M \rtimes G)=\sum_{(\phi) \in \operatorname{Hom}\left(\Gamma_{1}, G\right) / G} \chi_{\Gamma_{2}}^{\text {top }}\left(M^{\langle\phi\rangle} \rtimes C_{G}(\phi)\right) .
$$

In the proof of [20, Theorem 3.2], the second author demonstrated that $\chi_{\mathrm{ES}}(\widetilde{Q})=$ $\chi_{\text {top }}(Q)$, where $\widetilde{Q}$ denotes the inertia orbifold (see [2, Definition 2.49]). Note that this proof does not require the codimension-2 condition nor the hypotheses that $Q$ is effective or orientable. In [8, Corollary 3.8], the authors show that the inertia orbifold corresponds to the $\mathbb{Z}$-sectors. Hence, we have the relationship

$$
\chi_{\text {top }}(Q)=\chi_{\mathbb{Z}}^{\mathrm{ES}}(Q)=\chi_{\mathrm{ES}}(\tilde{Q}) .
$$

More generally,

$$
\chi_{\Gamma}^{\mathrm{top}}(Q)=\chi_{\Gamma \times \mathbb{Z}}^{\mathrm{ES}}(Q)
$$




\section{Wreath symmetric products}

In this section, we consider the wreath symmetric products of orbifolds presented as the quotient by the locally free action of a compact, connected Lie group. In Sections 5.1 and 5.2, we discuss the structure of wreath products of compact, connected Lie groups and the associated symmetric products; see Tamanoi [21] and Wang [25] for the case of $G$ finite. In Section 5.3 and Section 5.4, we use this structure and the properties of the $\Gamma$-Euler-Satake characteristics demonstrated above to derive formulas for the $\mathbb{Z}^{m}$-Euler-Satake characteristics of wreath symmetric products

Throughout this section, we let $G$ be a compact, connected Lie group acting smoothly, effectively and locally freely on the closed manifold $M$ and let $Q$ denote the orbifold presented by $M \rtimes G$. We let $G^{n}=\prod_{i=1}^{n} G$ denote the direct product with elements denoted $\mathbf{g}=\left(g_{1}, \ldots, g_{n}\right)$.

\subsection{Wreath products of compact groups}

Let $\mathcal{S}_{n}$ denote the symmetric group on a set of $n$ elements. Then $\mathcal{S}_{n}$ acts on $G^{n}$ by setting

$$
s(\mathbf{g})=\left(g_{s^{-1}(1)}, \ldots, g_{s^{-1}(n)}\right)
$$

for each $s \in \mathcal{S}_{n}$ and $\mathbf{g}=\left(g_{1}, \ldots, g_{n}\right) \in G^{n}$. Then the wreath product $G\left(\mathcal{S}_{n}\right)$ of $G$ by $\mathcal{S}_{n}$ is the semidirect product of $G^{n}$ by this action. In particular, we have

$$
(\mathbf{g}, s)(\mathbf{h}, t)=(\mathbf{g} s(\mathbf{h}), s t) \text { and }(\mathbf{g}, s)^{-1}=\left(s^{-1}\left(\mathbf{g}^{-1}\right), s^{-1}\right)
$$

for $\mathbf{g}, \mathbf{h} \in G^{n}$ and $s, t \in \mathcal{S}_{n}$.

5.1.1 Conjugacy classes The conjugacy classes of $G\left(\mathcal{S}_{n}\right)$ correspond to conjugacy classes of $G$ and conjugacy classes of $\mathcal{S}_{n}$. We can decompose each element $(\mathbf{g}, s) \in G\left(\mathcal{S}_{n}\right)$ into a product $(\mathbf{g}, s)=\prod_{j}\left(\mathbf{g}_{j}, s_{j}\right)$ of disjoint cycles corresponding to the cycle decomposition $s=\prod_{j} s_{j}$ in $\mathcal{S}_{n}$, where for each $j$, we define $\mathbf{g}_{j}$ to be the element of $G^{n}$ associated to $\mathbf{g}$ having nontrivial entries only in the positions $\left\{i_{1}, \ldots, i_{r}\right\}$ corresponding to $s_{j}=\left(i_{1} \cdots i_{r}\right)$. For each $j$, let $c_{j} \in G$ denote the cycle product associated to $s_{j}$, ie the product of the components of $\mathbf{g}_{j}$. See Tamanoi [21, page 129] or Wang [25, Section 1].

The following proposition, giving information about the conjugacy class of $(\mathbf{g}, s)$ in $G\left(\mathcal{S}_{n}\right)$, is stated in [21, Proposition 3-1] for $G$ finite. Notice that the outside product in Equation (11) is restricted to a finite set; with this modification, Tamanoi's proof by direct computation extends to the case of $G$ compact and connected. We let $G_{*}$ denote the set of conjugacy classes of $G$ and $(c)$ the conjugacy class of $c \in G$. 
Proposition 5.1 Let $(\mathbf{g}, s)=\prod_{j}\left(\mathbf{g}_{j}, s_{j}\right) \in G\left(\mathcal{S}_{n}\right)$ be a disjoint cycle decomposition of a fixed $(\mathbf{g}, s) \in G\left(\mathcal{S}_{n}\right)$ as above. For a fixed $j$ with $s_{j}=\left(i_{1} i_{2} \cdots i_{r}\right)$, define $\mathbf{d}_{j}=\left(g_{i_{1}}, g_{i_{2}} g_{i_{1}}, \ldots, g_{i_{r}} g_{i_{r-1}} \ldots g_{i_{1}}\right) \in G^{r}$ and $\mathbf{c}_{j}=\left(c_{j}, 1, \ldots, 1\right) \in G^{r}$ with $c_{j}=$ $g_{i_{1}} \cdots g_{i_{r}}$ as above. Treating $\left(\mathbf{g}_{j}, s_{j}\right)$ as an element of $G\left(\mathcal{S}_{r}\right)$ in the obvious way, we have

$$
\left(\mathbf{g}_{j}, s_{j}\right)=\left(\mathbf{d}_{j}, 1\right)\left(\mathbf{c}_{j}, s_{j}\right)\left(\mathbf{d}_{j}, 1\right)^{-1} \in G\left(\mathcal{S}_{r}\right) .
$$

Moreover, $\left(\mathbf{d}_{j}, 1\right)$ and $\left(\mathbf{d}_{k}, 1\right)$ commute for any $j \neq k$.

Let $G_{*}(\mathbf{g}, s)$ denote the collection of conjugacy classes $(c) \in G_{*}$ such that $c_{j} \in(c)$ for some $j$. For each $(c) \in G_{*}(\mathbf{g}, s)$ and each $r=1,2, \ldots, n$, let $m_{r}(c)$ denote the number of $r$-cycles $s_{j}$ of $s$ such that the corresponding $c_{j}$ is conjugate to $c$. We enumerate the corresponding $s_{j}$ and $\mathbf{c}_{j}$ as $s_{j((c), r)_{1}}, \ldots, s_{j((c), r)_{m_{r}(c)}}$ and $\mathbf{c}_{j_{1}}, \ldots, \mathbf{c}_{j_{m_{r}(c)}}$, which are now considered elements of $\mathcal{S}_{n}$ and $G\left(\mathcal{S}_{n}\right)$, respectively. Let $(\mathbf{d}, 1)=\prod_{j}\left(\mathbf{d}_{j}, 1\right) \in$ $G\left(\mathcal{S}_{r}\right)$. Then the cycle decomposition $(\mathbf{g}, s)=\prod_{j}\left(\mathbf{g}_{j}, s_{j}\right)$ of $(\mathbf{g}, s)$ induces the decomposition, up to conjugation,

$$
(\mathbf{g}, s) \sim(\mathbf{d}, 1)\left(\prod_{(c) \in G_{*}(\mathbf{g}, s)} \prod_{r=1}^{n} \prod_{i=1}^{m_{r}(c)}\left(\mathbf{c}_{j((c), r)_{i}}, s_{j((c), r)_{i}}\right)\right)(\mathbf{d}, 1)^{-1} \in G\left(\mathcal{S}_{n}\right) .
$$

Note that $G_{*}(\mathbf{g}, s)$ is finite so that the above product is defined.

Proof Equation (10) can be verified by a direct computation. Then as the $\left(\mathbf{g}_{j}, s_{j}\right)$ commute, Equation (11) follows as we are taking the product over all possible cycle lengths.

It follows from Proposition 5.1 that for each cycle $s_{j}$, the conjugacy class of the corresponding $c_{j}$ is uniquely determined. Hence, we have the following.

Definition 5.2 Fix $(\mathbf{g}, s) \in G\left(\mathcal{S}_{n}\right)$. With the notation as in Proposition 5.1, there is a partition-valued function $\rho: G_{*} \rightarrow \mathcal{P}$ called the type of $(\mathbf{g}, s)$, denoted $\rho((c))=$ $\left(m_{r}(c)\right)$ for $r=1, \ldots, n$. Here, $\mathcal{P}$ denotes the set of partitions of nonnegative integers. As $s \in \mathcal{S}_{n}$ is decomposed into cycles,

$$
\sum_{r=1}^{n} \sum_{(c) \in G_{*}} r m_{r}(c)=n
$$

In particular, $t_{c}=\sum_{r=1}^{n} r m_{r}(c) \leq n$ is finite. If we set $m_{r}=\sum_{(c) \in G_{*}} m_{r}(c)$, then $\sum_{r=1}^{n} r m_{r}=n$ so that the $m_{r}$ determine a partition of $n$. 
By Proposition 5.1, the conjugacy classes of $G\left(\mathcal{S}_{n}\right)$ are parameterized by types, generalizing to wreath products the well-known fact that the conjugacy classes of $\mathcal{S}_{n}$ are determined by the structure of their cycle decomposition.

5.1.2 Centralizers We will now study the structure of the centralizer of an element $(\mathbf{g}, s)$ of the wreath product $G\left(\mathcal{S}_{n}\right)$ up to conjugation by $G\left(\mathcal{S}_{n}\right)$. By Theorem 5.3, we have that $C_{G\left(\mathcal{S}_{n}\right)}(\mathbf{g}, s)$ can be decomposed as a product of wreath products.

Let $(\mathbf{g}, s)=\prod_{j}\left(\mathbf{g}_{j}, s_{j}\right) \in G\left(\mathcal{S}_{n}\right)$ be a disjoint cycle decomposition of $(\mathbf{g}, s)$. If $(\mathbf{h}, t) \in G\left(\mathcal{S}_{n}\right)$ commutes with $(\mathbf{g}, s)$, then conjugation by $(\mathbf{h}, t)$ permutes the $m_{r}(c)$

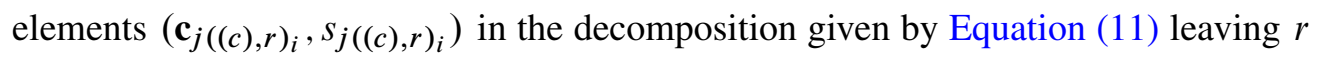
and $(c)$ fixed. It therefore induces a homomorphism

$$
p: C_{G\left(\mathcal{S}_{n}\right)}(\mathbf{g}, s) \longrightarrow \prod_{(c) \in G_{*}(\mathbf{g}, s)} \prod_{r=1}^{n} \mathcal{S}_{m_{r}(c)},
$$

where $\mathcal{S}_{m_{r}(c)}$ as usual denotes the permutation group on $m_{r}(c)$ elements.

With this, the following important description of the centralizer of $(\mathbf{g}, s)$ in $G\left(\mathcal{S}_{n}\right)$ generalizes to the case of $G$ compact, connected with identical proof; see Tamanoi [21, Lemma 3-4 and Theorem 3-5] and Wang [25, Lemma 2].

Theorem 5.3 Let $(\mathbf{g}, s)=\prod_{j}\left(\mathbf{g}_{j}, s_{j}\right) \in G\left(\mathcal{S}_{n}\right)$ be a disjoint cycle decomposition. For a fixed $j$ where $s_{j}$ has length $r$, the centralizer $C_{G\left(\mathcal{S}_{r}\right)}\left(\mathbf{g}_{j}, s_{j}\right)$ of $\left(\mathbf{g}_{j}, s_{j}\right)$ treated as an element of $G\left(\mathcal{S}_{r}\right)$ in the obvious way is given by the product

$$
C_{G\left(\mathcal{S}_{r}\right)}\left(\mathbf{g}_{j}, s_{j}\right)=C_{G}\left(c_{j}\right)\left\langle a_{r, c_{j}}\right\rangle
$$

where $c_{j}$ is the cycle product of $\mathbf{g}_{j}$ and $\mathbf{c}_{j}=\left(c_{j}, 1, \ldots, 1\right) \in G^{r}$ as above, $a_{r, c_{j}}=$ $\left(\mathbf{c}_{j},(12 \cdots r)\right)$, and $C_{G}\left(c_{j}\right)$ is considered a subgroup of $G\left(\mathcal{S}_{r}\right)$ via the diagonal map. By $C_{G}(c)\left\langle a_{r, c}\right\rangle$, we mean the subgroup of $G\left(\mathcal{S}_{r}\right)$ generated by $C_{G}(c)$ and $a_{r, c}$. Then

$$
C_{G\left(\mathcal{S}_{n}\right)}(\mathbf{g}, s) \cong \prod_{(c) \in G_{*}(\mathbf{g}, s)} \prod_{r=1}^{n}\left[C_{G}(c)\left\langle a_{r, c}\right\rangle\right]\left(\mathcal{S}_{m_{r}(c)}\right),
$$

where $\left[C_{G}(c)\left\langle a_{r, c}\right\rangle\right]\left(\mathcal{S}_{m_{r}(c)}\right)$ is a wreath product and $m_{r}(c)$ again denotes the number of $r$-cycles in the cycle decomposition of $(\mathbf{g}, s)$ with cycle product in the conjugacy class $(c)$. Note that $a_{r, c}^{r}=((c, \ldots c), 1)$ and $\left[a_{r, c}, C_{G}(c)\right]=1$. The isomorphism is induced by the conjugation given in Equation (11).

Hence, the centralizer of an element $(\mathbf{g}, s) \in G\left(\mathcal{S}_{n}\right)$ can be decomposed into a product of centralizers according to the cycle decomposition of $s \in \mathcal{S}_{n}$. 


\subsection{Actions of wreath products on manifolds}

Let $M$ be a $G$-manifold, and consider the action of $G\left(\mathcal{S}_{n}\right)$ on $M^{n}$ given by

$$
(\mathbf{g}, s)\left(x_{1}, \ldots, x_{n}\right)=\left(g_{1} x_{s^{-1}(1)}, \ldots, g_{n} x_{s^{-1}(n)}\right) .
$$

We let $M G\left(\mathcal{S}_{n}\right)$ denote the orbifold presented by the groupoid $M^{n} \rtimes G\left(\mathcal{S}_{n}\right)$, called the wreath symmetric product of $M \rtimes G$. When $n=1$, note that $M \rtimes G\left(\mathcal{S}_{1}\right)=M \rtimes G$. In this section, we describe the structure of the fixed point sets $\left(M^{n}\right)^{\langle(\mathrm{g}, s)\rangle}$ for a fixed $(\mathbf{g}, s) \in G\left(\mathcal{S}_{n}\right)$. We continue to follow Tamanoi [21] and Wang [25].

We make repeated use of the following general fact. Suppose $K$ is a compact, connected Lie group acting locally freely, effectively and smoothly on the smooth manifold $Z$ in such a way that $Z \rtimes K$ is Morita equivalent to an orbifold groupoid. By Adem and Ruan [2, Theorem 5.3], the standard cohomology of the inertia orbifold is given by

$$
H^{*}\left((Z \rtimes K)^{\mathbb{Z}}\right)=\bigoplus_{(k) \in t_{Z ; K}} H^{*}\left(Z^{\langle k\rangle} \rtimes C_{K}(k)\right),
$$

where $t_{Z ; K}$ denotes the set of conjugacy classes in $K$ with nonempty fixed point set in $Z$. It follows that the delocalized cohomology (ie, the equivariant cohomology of the inertia orbifold) is isomorphic to the complexified orbifold $K$-theory.

If $K$ and $L$ are compact, connected Lie groups such that $L$ acts on $K$ and $K \rtimes L$ acts on the manifold $Z$, then

$$
H^{*}(Z \rtimes(K \rtimes L)) \cong H^{*}(Z)^{K \rtimes L} \cong H^{*}(Z \rtimes L)^{K}
$$

where the latter two expressions indicate the invariant cohomology.

We now return to the case of a wreath symmetric product. We begin by studying the special case of $(\mathbf{g}, s)=((g, 1, \ldots, 1), \tau) \in G\left(\mathcal{S}_{n}\right)$ with $\tau=(1,2, \ldots, n) \in \mathcal{S}_{n}$. Given Proposition 5.1, the proofs of Wang [25, Lemmas 4 and 5] generalize to the case of $G$ compact and connected, yielding the following.

Proposition 5.4 Suppose that $a=((g, 1, \ldots, 1), \tau) \in G\left(\mathcal{S}_{n}\right)$ for some $g \in G$ with $\tau=(1,2, \ldots, n) \in \mathcal{S}_{n}$. Then

$$
\left(M^{n}\right)^{\langle a\rangle}=\left\{(x, \ldots, x) \in M^{n}: x=g x\right\} .
$$

The group $C_{G\left(\mathcal{S}_{n}\right)}(a)$ is isomorphic to $C_{G}(g)\langle a\rangle$, and the action of $C_{G}(g)\langle a\rangle$ on $\left(M^{n}\right)^{\langle a\rangle}$ can be identified via the diagonal map with the action of $C_{G}(g)$ on $M^{\langle g\rangle}$ together with the trivial a-action. Therefore, by Equation (13), we have

$$
H^{*}\left(\left(M^{n}\right)^{\langle a\rangle} \rtimes C_{G\left(\mathcal{S}_{n}\right)}(a)\right) \cong H^{*}\left(M^{\langle g\rangle}\right)^{C_{G}(g)} .
$$


Using the decomposition given in Proposition 5.1, it follows that $G\left(\mathcal{S}_{n}\right)$ acts locally freely on $M^{n}$ if and only if $G$ acts locally freely on $M$. Hence, $M \rtimes G$ is Morita equivalent to an orbifold groupoid if and only if the same holds for $M^{n} \rtimes G\left(\mathcal{S}_{n}\right)$. With this, we can determine the delocalized equivariant cohomology of $\operatorname{MG}\left(\mathcal{S}_{n}\right)$, that is, the standard de Rham cohomology (via $G$-invariant differential forms) of the inertia orbifold $\widetilde{M G\left(\mathcal{S}_{n}\right)_{\mathbb{Z}}}$ of $M G\left(\mathcal{S}_{n}\right)$ in the case that $M \rtimes G$ is Morita equivalent to an orbifold groupoid. This result is a straightforward generalization of results of Tamanoi in $[21 ; 22]$.

Proposition 5.5 For a fixed $(\mathbf{g}, s) \in G\left(\mathcal{S}_{n}\right)$ with nonempty fixed-point set in $M^{n}$, the groupoid $\left(M^{n}\right)^{\langle(\mathbf{g}, s)\rangle} \rtimes C_{G\left(\mathcal{S}_{n}\right)}((\mathbf{g}, s))$ is given as follows. First, let

$$
Y^{m_{r}(c)}=\left(M^{\langle c\rangle}\right)^{m_{r}(c)} \rtimes\left(C_{G}(c)\right)^{m_{r}(c)},
$$

and let $\mathcal{S}_{m_{r}(c)}$ act on $Y^{m_{r}(c)}$ by permuting the coordinates of $\left(M^{\langle c\rangle}\right)^{m_{r}(c)}$.

It follows by Equation (13) that

$$
H^{*}\left(\left(M^{n}\right)^{\langle a\rangle} \rtimes C_{G\left(\mathcal{S}_{n}\right)}(a)\right) \cong \bigotimes_{(c) \in t_{M ; G}} \bigotimes_{r=1}^{n} H^{*}\left(\left(M^{\langle c\rangle}\right)^{m_{r}(c)} \rtimes C_{G}(c)^{m_{r}(c)}\right)^{\mathcal{S}_{m_{r}(c)}},
$$

where $t_{c}=\sum_{r} r m_{r}(c) \leq n$ and the factors where $m_{r}(c)=0$ correspond to points with trivial action.

Remark 5.6 The above results imply that the conjugacy classes of $G\left(\mathcal{S}_{n}\right)$ with nonempty fixed point sets in $M^{n}$ are parameterized by the finite set $t_{M ; G}$ of conjugacy classes $(c) \in G_{*}$ with nonempty fixed point sets and their types $m_{r}(c)$ with $r=1, \ldots, n$. In particular, let $t_{M ; G}=\left\{\left(c_{1}\right), \ldots,\left(c_{N}\right)\right\}$. The set $t_{M^{n} ; G\left(\mathcal{S}_{n}\right)}=\left\{(a) \in G\left(\mathcal{S}_{n}\right)\right.$ : $\left.\left(M^{n}\right)^{\langle a\rangle} \neq \varnothing\right\}$ is parameterized by the $m_{r}\left(c_{k}\right)$ with $r=1, \ldots, n$ and $k=1, \ldots N$. We emphasize the equalities $\sum_{(c) \in t_{M ; G}} \sum_{r} r m_{r}(c)=n$ and $\sum_{r} r m_{r}=n$ from Definition 5.2 , which play an important role in the sequel.

By the above observations, when $M / G$ and hence $M G\left(\mathcal{S}_{n}\right)$ is an orbifold, the inertia orbifold ${\widetilde{\left(M G\left(\mathcal{S}_{n}\right)\right)_{\mathbb{Z}}}}_{\mathbb{M}}$ of $M G\left(\mathcal{S}_{n}\right)$ is presented by the groupoid

$$
\begin{aligned}
\mathbb{I M G}_{n} & =\coprod_{((\mathbf{g}, s)) \in t_{M^{n} ; G\left(\mathcal{S}_{n}\right)}}\left(M^{n}\right)^{\langle(\mathbf{g}, s)\rangle} \rtimes C_{G\left(\mathcal{S}_{n}\right)}(\mathbf{g}, s) \\
& =\coprod_{m_{r}(c)} \prod_{r=1}^{n} \prod_{(c) \in t_{M ; G}}\left(\left(M^{\langle c\rangle}\right)^{m_{r}(c)} \rtimes\left(C_{G}(c)\left\langle a_{r, c}\right\rangle\right)^{m_{r}(c)}\right) \rtimes \mathcal{S}_{m_{r}(c)} .
\end{aligned}
$$


It follows that

$$
\begin{aligned}
H^{*}\left(\widetilde{\left(M G\left(\mathcal{S}_{n}\right)\right)_{\mathbb{Z}}}\right) & =\bigoplus_{((\mathbf{g}, s)) \in t_{M^{n} ; G\left(\mathcal{S}_{n}\right)}} H^{*}\left(\left(M^{n}\right)^{\langle(\mathbf{g}, s)\rangle} \rtimes C_{G\left(\mathcal{S}_{n}\right)}(\mathbf{g}, s)\right) \\
& \cong \bigoplus_{m_{r}(c)} \bigotimes_{r=1}^{n} \bigotimes_{(c) \in t_{M} ; G} H^{*}\left(\left(M^{\langle c\rangle}\right)^{m_{r}(c)} \rtimes\left(C_{G}(c)^{m_{r}(c)} \rtimes \mathcal{S}_{m_{r}(c)}\right)\right) \\
& \cong \bigoplus_{m_{r}(c)} \bigotimes_{r=1}^{n} \bigotimes_{(c) \in t_{M} ; G} H^{*}\left(\left(M^{\langle c\rangle}\right)^{m_{r}(c)} \rtimes C_{G}(c)^{m_{r}(c)}\right)^{\mathcal{S}_{m_{r}(c)}} .
\end{aligned}
$$

\subsection{Generalizations of MacDonald's formulas}

In this subsection, we prove Theorem 5.8 which generalizes MacDonald's formulas in MacDonald [13] and Wang [25, Theorem 5] to the context of an orbifold given by a quotient by a compact, connected Lie group acting smoothly and locally freely. This formula will serve as the base case in the induction proof of Theorem 5.11.

First, we derive the following formula for the Euler-Satake characteristic of a wreath symmetric product $M G\left(\mathcal{S}_{n}\right)$ as defined in Section 5.2.

Proposition 5.7 For a quotient orbifold $Q$ presented by $M \rtimes G$,

$$
\chi_{\mathrm{ES}}\left(M G\left(\mathcal{S}_{n}\right)\right)=\frac{1}{n !}\left(\chi_{\mathrm{ES}}(Q)\right)^{n} .
$$

Proof By using the slice theorem, it is straightforward to show that the natural map $G^{n} x \mapsto G\left(\mathcal{S}_{n}\right) x$ from the orbifold presented by $M^{n} \rtimes G^{n}$ to the orbifold $M G\left(\mathcal{S}_{n}\right)$ is an orbifold cover of with $n$ ! sheets.

Now, note that $M^{n} \rtimes G^{n}=(M \rtimes G)^{n}$ so that by Theorem 2.3, $\chi_{\mathrm{ES}}\left(M^{n} \rtimes G^{n}\right)=$ $\left(\chi_{\mathrm{ES}}(Q)\right)^{n}$. Then the result follows from Lemma 2.2.

Theorem 5.8 Let $G$ be a compact, connected Lie group acting smoothly, effectively and locally freely on the closed manifold $M$. Let $Q$ denote the orbifold presented by $M \rtimes G$ and $M G\left(\mathcal{S}_{n}\right)$ denote the wreath symmetric product.

(1) The de Rham cohomology $H^{*}\left(M G\left(\mathcal{S}_{n}\right)\right)$ of $G\left(\mathcal{S}_{n}\right)$-invariant differential forms on $M^{n}$ satisfies the MacDonald formulas

$H^{*}\left(M G\left(\mathcal{S}_{n}\right)\right) \cong \mathcal{S} \mathcal{P}^{n}\left[H^{*}(Q)\right]$ and $\sum_{n \geq 0} \operatorname{dim}\left[H^{*}\left(M G\left(\mathcal{S}_{n}\right)\right)\right] q^{n}=\frac{1}{(1-q)^{\operatorname{dim}\left[H^{*}(Q)\right]}}$

where $\mathcal{S P}$ denotes the symmetric product and $\operatorname{dim}[W]=\left|\operatorname{dim}\left[W^{\mathrm{ev}}\right] \otimes \mathbb{C}\right|-$ $\left|\operatorname{dim}\left[W^{\text {odd }}\right] \otimes \mathbb{C}\right|$ denotes the Euler characteristic of the complex $W$. 
(2) The delocalized equivariant cohomology $H_{\mathbb{Z}}^{*}\left(M G\left(\mathcal{S}_{n}\right)\right)$ satisfies the MacDonald formula

$$
\left.\bigoplus_{n \geq 0} H_{\mathbb{Z}}^{*}\left(M G\left(\mathcal{S}_{n}\right)\right) q^{n} \cong \mathcal{S P}\left(\bigoplus_{n \geq 0} H^{*}(\widetilde{(M / G)})_{\mathbb{Z}}\right) q^{n}\right)
$$

As well, we have the dimension formula

$$
\sum_{n \geq 0} \operatorname{dim}\left[H_{\mathbb{Z}}^{*}\left(M G\left(\mathcal{S}_{n}\right)\right)\right] q^{n}=\prod_{n \geq 1} \frac{1}{\left(1-q^{n}\right)^{\operatorname{dim}\left(H_{\mathbb{Z}}^{*}(M \rtimes G)\right)}} .
$$

Proof See Wang [25, Section 6], Zhou [29] and Tamanoi [21, Section 4].

The $K$-theory of the crossed product algebra $C(M) \rtimes G$ is isomorphic to the equivariant $K$-theory of $M$ (see eg Blackadar [4]), which coincides with the orbifold $K$-theory of $Q$ (see Adem, Leida and Ruan [1, Definition 3.8]). For a quotient orbifold $Q$, recall that $\chi_{\mathbb{Z}^{2}}^{\mathrm{ES}}(Q)$ coincides with the stringy orbifold Euler characteristic and hence the Euler characteristic of the orbifold $K$-theory of $Q$. Hence

$$
\chi_{\mathbb{Z}^{2}}^{\mathrm{ES}}(Q)=\operatorname{dim} K^{0}(C(M) \rtimes G) \otimes \mathbb{C}-\operatorname{dim} K^{1}(C(M) \rtimes G) \otimes \mathbb{C} .
$$

As the (complexified) $K$-theory is isomorphic to the delocalized cohomology, we have the following.

Corollary 5.9 With $M$ and $G$ as in Theorem 5.8,

$$
\sum_{n \geq 0} \chi_{\mathbb{Z}^{2}}^{\mathrm{ES}}\left(M G\left(\mathcal{S}_{n}\right)\right) q^{n}=\sum_{n \geq 0} \operatorname{dim}\left[K^{*}\left(M G\left(\mathcal{S}_{n}\right)\right)\right] q^{n}=\prod_{n \geq 1} \frac{1}{\left(1-q^{n}\right)^{\operatorname{dim}\left(K^{*}(M \rtimes G)\right)}} .
$$

\subsection{Wreath symmetric products and product formulas}

In this section, we prove a product formula for the $\mathbb{Z}^{m}$-Euler characteristics that extends Tamanoi [21, Theorem A] to the case of a quotient by a compact, connected group. For simplicity, we abbreviate $\chi_{\mathbb{Z}^{m}}^{\text {top }}$ as $\chi_{(m)}$ and $\chi_{\mathbb{Z}^{m}}^{\mathrm{ES}}$ as $\chi_{(m)}^{\mathrm{ES}}$.

The following result is proven as in [21, Lemma 4-1].

Lemma 5.10 Let $L$ be a compact Lie group, $K$ a closed, connected subgroup, and suppose there is an $a \in L$ such that $\langle a\rangle \cap K=\left\langle a^{r}\right\rangle$ and $a \in C_{L}(K)$. Let $M$ be a $K$-manifold on which $K$ acts smoothly, effectively and locally freely, and suppose $a$ acts trivially on $M$. Then for each $m \geq 0$,

$$
\chi_{(m)}(M \rtimes K\langle a\rangle)=r^{m} \chi_{(m)}(M \rtimes K) .
$$


Theorem 5.11 Let $G$ be a compact, connected Lie group acting smoothly, effectively and locally freely on the closed manifold $M$, and let $Q$ be the orbifold presented by $M \rtimes G$. Then

$$
\sum_{n=0}^{\infty} \chi_{\mathrm{ES}}\left(M G\left(\mathcal{S}_{n}\right)\right) q^{n}=\exp \left(q \chi_{\mathrm{ES}}(Q)\right)
$$

For $m \geq 0$,

$$
\sum_{n=0}^{\infty} \chi_{(m)}\left(M G\left(\mathcal{S}_{n}\right)\right) q^{n}=\prod_{j_{1}, \ldots, j_{m} \geq 1}\left[\left(1-q^{j_{1} j_{2} \cdots j_{m}}\right)^{j_{2} j_{3}^{2} \cdots j_{m-1}^{m-2} j_{m}^{m-1}}\right]^{-\chi(m)}(Q) .
$$

Noting that $J_{r, m}=\sum_{j_{1} \cdots j_{m}=r} j_{2} j_{3}^{2} \cdots j_{m-1}^{m-2} j_{m}^{m-1}$ is the number of subgroups of index $r$ in $\mathbb{Z}^{m}$, Equation (17) can be rewritten as

$$
\sum_{n=0}^{\infty} \chi_{(m)}\left(M G\left(\mathcal{S}_{n}\right)\right) q^{n}=\prod_{r \geq 1}\left[\left(1-q^{r}\right)^{J_{r, m}}\right]^{-\chi_{(m)}(Q)} .
$$

Proof Equation (16) follows by applying Proposition 5.7. The proof of Equation (17) and Equation (18) is by induction on $m$. As $\chi_{(0)}(Q)=\chi_{\text {top }}(Q)$ and $\chi_{(1)}(Q)=$ $\chi_{\text {top }}\left(\widetilde{Q}_{\mathbb{Z}}\right)$, the cases $m=0$ and $m=1$ of Equation (17) corresponds to

$$
\sum_{n=0}^{\infty} \chi_{\mathrm{top}}\left(M G\left(\mathcal{S}_{n}\right)\right) q^{n}=(1-q)^{-\chi_{\mathrm{top}}(Q)}
$$

and

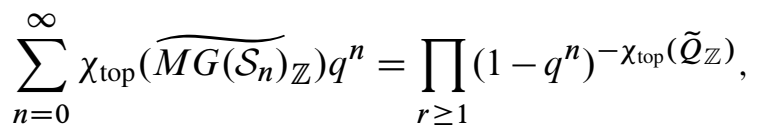

both following directly from Theorem 5.8.

For $m>1$, we have that

$$
\begin{aligned}
\sum_{n \geq 0} q^{n} \chi_{(m)}\left(M G\left(\mathcal{S}_{n}\right)\right) & \\
& =\sum_{n \geq 0} q^{n} \chi_{(m-1)}\left(\widehat{\left(\overline{\left.M G\left(\mathcal{S}_{n}\right)\right)_{\mathbb{Z}}}\right)}\right. \\
& =\sum_{n \geq 0} q^{n} \sum_{((\mathbf{g}, s)) \in t_{M^{n} ; G\left(\mathcal{S}_{n}\right)}} \chi_{(m-1)}\left(\left(M^{n}\right)^{\langle(\mathbf{g}, s)\rangle} \rtimes C_{G\left(\mathcal{S}_{n}\right)}(\mathbf{g}, s)\right) \\
& =\sum_{n \geq 0} q^{n} \chi_{(m-1)}\left(\coprod_{m_{r}(c)} \prod_{r} \prod_{(c)}\left(\left(M^{\langle c\rangle}\right)^{m_{r}(c)} \rtimes\left(\left[C_{G}(c)\left\langle a_{r, c}\right\rangle\right]^{m_{r}(c)} \rtimes \mathcal{S}_{m_{r}(c)}\right)\right)\right),
\end{aligned}
$$


where the union and products are over all $m_{r}(c) \geq 0$ for $(c) \in t_{M} ; G, r \geq 1$ subject to the constraint that $\sum_{r, c} r m_{r}(c)=n$ and $(c) \in t_{M ; G}$. Applying Corollary 4.4, we have

$$
\begin{aligned}
\sum_{n \geq 0} q^{n} \chi_{(m)}\left(M G\left(\mathcal{S}_{n}\right)\right) & \\
& =\sum_{n \geq 0} q^{n} \sum_{m_{r}(c)} \prod_{r} \prod_{(c)} \chi_{(m-1)}\left(\left(M^{\langle c\rangle}\right)^{m_{r}(c)} \rtimes\left(\left[C_{G}(c)\left\langle a_{r, c}\right\rangle\right]^{m_{r}(c)} \rtimes \mathcal{S}_{m_{r}(c)}\right)\right), \\
& =\sum_{n \geq 0} q^{n} \sum_{m_{r}(c)} \prod_{r} \prod_{(c)} \chi_{(m-1)}\left(\mathcal{S P}^{m_{r}(c)}\left(M^{\langle c\rangle}\right) \rtimes\left(C_{G}(c)\left\langle a_{r, c}\right\rangle\right)\right),
\end{aligned}
$$

where the symmetric product of a crossed product groupoid $X=K \rtimes L$ is defined in a similar way to the symmetric product of a vector space; that is, $\mathcal{S P}^{m}[X]=$ $K^{m} \rtimes\left(L^{m} \rtimes \mathcal{S}_{m}\right)$. In other words, the symmetric product is the wreath product.

As in the proof of [21, Equation (4-1)], the above formula implies that if we again set $m_{r}=\sum_{c} m_{r}(c)$, we have

$$
\begin{aligned}
\sum_{n \geq 0} q^{n} \chi_{(m)}\left(M G\left(\mathcal{S}_{n}\right)\right) & =\sum_{n \geq 0} q^{n} \chi_{(m-1)}\left({\widehat{\left(M G\left(\mathcal{S}_{n}\right)\right)_{\mathbb{Z}}}}\right) \\
& =\sum_{n \geq 0} q^{n} \sum_{m_{r}} \prod_{r} \chi_{(m-1)}\left(\mathcal{S P}{ }^{m_{r}}[\mathbb{M M G} r]\right),
\end{aligned}
$$

where now the sum is over all $m_{r} \geq 0$ and $r \geq 1$ such that $\sum_{r} r m_{r}=n$, and $\mathcal{S P}^{m_{r}}=\bigoplus_{(c)} \mathcal{S P}^{m_{r}(c)}$. Note that the groupoid $\mathbb{I M G} \mathbb{G}_{r}$ is defined in Equation (14). Hence, this is equal to

$$
\sum_{m_{r}} \prod_{r} q^{r m_{r}} \chi_{(m-1)}\left(\mathcal{S} \mathcal{P}^{r m_{r}}\left[\mathbb{M M G} \mathbb{G}_{r}\right]\right)
$$

with no constraints on $m_{r} \geq 0$. As $\chi_{(m-1)}$ is a multiplicative character, this is equal to

$$
\prod_{r} \sum_{m_{r}} q^{r m_{r}} \chi_{(m-1)}\left(\mathcal{S} \mathcal{P}^{r m_{r}}\left[\mathbb{I M} \mathbb{G}_{r}\right]\right)
$$

Now, by the inductive hypothesis,

$$
\begin{aligned}
\sum_{n \geq 0} q^{n} \chi_{(m)} & \left(M G\left(\mathcal{S}_{n}\right)\right) \\
= & \prod_{r}\left(\prod_{r_{1}, \ldots, r_{m-1} \geq 1}\left(1-\left(q^{r}\right)^{r_{1}, \ldots, r_{m-1}}\right)^{r_{2} r_{3}^{2} \ldots r_{m-1}^{m-2}}\right)^{-\chi_{(m-1)}\left(\mathbb{M} \mathbb{G}_{r}\right)} .
\end{aligned}
$$

By Lemma 5.10, Theorem 5.3 and Proposition 5.5,

$$
\chi_{(m-1)}\left(\mathbb{I M G} \mathbb{G}_{r}\right)=r^{m-1} \chi_{(m-1)}\left({\widetilde{(M \rtimes G})_{\mathbb{Z}}} .\right.
$$


Combining this with Equation (19), we have

$\sum_{n \geq 0} q^{n} \chi_{(m)}\left(M G\left(\mathcal{S}_{n}\right)\right)=\left[\prod_{r, r_{1}, \ldots, r_{m-1} \geq 1}\left(1-q^{r_{1}, \ldots, r_{m-1} r}\right)^{r_{2} r_{3}^{2} \ldots r_{m-1}^{m-2} r^{m-1}}\right]^{-\chi(m)}(Q)$

completing the proof.

An application of Equation (9) allows us to express Theorem 5.11 in terms of the $\mathbb{Z}^{m}$-Euler-Satake characteristics as follows.

Corollary 5.12 Let $G$ be a compact, connected Lie group acting smoothly, effectively and locally freely on the closed manifold $M$, and let $Q$ be the orbifold presented by $M \rtimes G$. Then

$$
\sum_{n=0}^{\infty} \chi_{(m)}^{\mathrm{ES}}\left(M G\left(\mathcal{S}_{n}\right)\right) q^{n}= \begin{cases}\exp \left(q \chi_{\mathrm{ES}}(Q)\right) & m=0 \\ \prod_{r \geq 1}\left[\left(1-q^{r}\right)^{J_{r, m-1}}\right]^{-\chi_{(m)}^{\mathrm{ES}}(Q)} & m>0 .\end{cases}
$$

\section{Generalized Hodge numbers}

In this section, we prove Theorem 6.4, yielding a product formula for the orbifold Hodge numbers of a quotient by a compact, complex, connected Lie group $G$. See Wang and Zhou [26] for the case of $G$ finite. Note that for special choices of the parameters $x$ and $y$ in Theorem 6.4, we obtain interesting geometric invariants; see the note after Definition 6.1.

To derive our product formula for the shifted Hodge numbers, we will use the product formula for the case of the Hodge-Poincaré numbers and make the necessary modifications that account for the degree shifting. We assume that $G$ is a compact complex connected Lie group acting effectively, holomorphically and locally freely on the compact complex manifold $M$, and $Q$ is the complex orbifold presented by $M \rtimes G$. We use here the complex standard cohomology of differential forms $H^{s, t}(Q)$. The following definition follows [26, page 157].

Definition 6.1 Let the compact, complex, connected Lie group $G$ act effectively, locally freely and holomorphically on the complex manifold $M$. We define the standard orbifold Hodge polynomials $H(M \rtimes G ; x, y)$, the generating function of bigraded 
spaces and $h(M \rtimes G ; x, y)$, its graded dimension, by

and

$$
H(M \rtimes G ; x, y)=\sum_{s, t \geq 0} H^{s, t}(M \rtimes G) x^{s} y^{t}
$$

$$
h(M \rtimes G ; x, y)=\sum_{s, t \geq 0}\left|\operatorname{dim}_{\mathbb{C}}\left[H^{s, t}(M \rtimes G)\right]\right| x^{s} y^{t},
$$

where $H^{s, t}(M \rtimes G)$ are the standard Dolbeault cohomology groups of the complex orbifold $M \rtimes G$. We define the standard delocalized orbifold Hodge polynomials $H_{D}(M \rtimes G ; x, y)$ and $h_{D}(M \rtimes G ; x, y)$ by

$$
H_{D}(M \rtimes G ; x, y)=\sum_{s, t \geq 0} H^{s, t}\left(\widetilde{Q}_{\mathbb{Z}}\right) x^{s} y^{t}
$$

and

$$
h_{D}(M \rtimes G ; x, y)=\sum_{s, t \geq 0} \mid \operatorname{dim}_{\mathbb{C}}\left[H^{s, t}\left(\widetilde{Q}_{\mathbb{Z}}\right) \mid x^{s} y^{t} .\right.
$$

For example, it is well known that the Hirzebruch genus of $M \rtimes G$ is given by $h(M \rtimes G, y,-1)$, the (topological) Euler characteristic by $h(M \rtimes G,-1,-1)$ and the signature by $h(M \rtimes G, 1,-1)$.

In the remaining definitions, we will need to consider the grading shift. In this context, the degree shifting number in Adem, Leida and Ruan [1, Section 4.2] corresponds to that of Zaslow [28], which we now recall.

Fix $c \in G$, and let $M_{1}^{\langle c\rangle}, \ldots, M_{N_{c}}^{\langle c\rangle}$ be the connected components of $M^{\langle c\rangle}$. We let $F_{j}^{c}$ denote the shift number associated to each $M_{j}^{\langle c\rangle}, j=1, \ldots, N_{c}$, obtained by summing over the angles associated to the action of $G$ on the tangent to $M_{j}^{\langle c\rangle}$. Then for $0 \leq p, q \leq \operatorname{dim}_{\mathbb{C}} Q$, the Chen-Ruan Dolbeault cohomology groups are given by

$$
H_{\mathrm{CR}}^{p, q}(M \rtimes G)=\bigoplus_{(c) \in t_{M ; G}} \bigoplus_{j=1}^{N_{c}} H^{p-F_{j}^{c}, q-F_{j}^{c}}\left(M_{j}^{\langle c\rangle} \rtimes C_{G}(c)\right) .
$$

The following definitions follow Wang and Zhou [26, page 156].

Definition 6.2 Let the compact, complex, connected Lie group $G$ act locally freely and holomorphically on the compact, complex manifold $M$ and suppose each of the shift numbers $F_{j}^{c}$ are integers. Define the shifted delocalized orbifold Hodge polynomials $h_{\mathrm{CR}}(M \rtimes G ; x, y)$ by

$$
h_{\mathrm{CR}}(M \rtimes G ; x, y)=\sum_{s, t \geq 0} \mid \operatorname{dim}\left[H_{\mathrm{CR}}^{s, t}(M \rtimes G) \mid x^{s} y^{t} .\right.
$$


Also define the shifted delocalized orbifold Hodge numbers $\mathcal{H}^{p, q}(M \rtimes G)$ of $M \rtimes G$ for $0 \leq p, q \leq \operatorname{dim}_{\mathbb{C}} Q$ by

$$
\mathcal{H}^{p, q}(M \rtimes G)=\sum_{(c) \in t_{M} ; G} \sum_{j=1}^{N_{c}} h_{j}^{p-F_{j}^{c}, q-F_{j}^{c}}(c),
$$

where

$$
h_{j}^{p, q}(c)=\operatorname{dim}_{\mathbb{C}}\left[H^{p, q}\left(M_{j}^{\langle c\rangle} \rtimes C_{G}(c)\right)\right]=(-1)^{p+q} \operatorname{dim}_{\mathbb{C}}\left[H^{p, q}\left(M_{j}^{\langle c\rangle} \rtimes C_{G}(c)\right)\right],
$$

for $j=1, \ldots, N_{g}, p, q \geq 0$.

To derive our product formula, we will use the following, which follows from general results on graded vector spaces (see Wang and Zhou [26, Theorem 3.1]).

Proposition 6.3 Let $N$ be a compact, complex manifold and let $K$ be a compact complex, connected Lie group acting effectively, holomorphically and locally freely on $N$ with integer shifts.

(1) The standard delocalized orbifold Hodge polynomials $H_{D}$ satisfy

$$
\bigoplus_{n \geq 0} H_{D}\left(N^{n} \rtimes\left(K^{n} \rtimes \mathcal{S}_{n}\right) ; x, y\right) q^{n}=\mathcal{S P}\left[\bigoplus_{r>0} H_{D}(N \rtimes K ; x, y) q^{r}\right],
$$

where $\mathcal{S P}$ again denotes the symmetric product algebra.

(2) The standard delocalized orbifold Hodge polynomials $h_{D}$ satisfy

$$
\sum_{n \geq 0} h_{D}\left(N^{n} \rtimes\left(K^{n} \rtimes \mathcal{S}_{n}\right) ; x, y\right) q^{n}=\prod_{n, s, t} \frac{1}{\left(1-x^{s} y^{t} q^{n}\right)^{h_{D}^{s, t}(N \rtimes K)}},
$$

where $\quad h_{D}^{s, t}(N \rtimes K)=\operatorname{dim}_{\mathbb{C}}\left(H_{D}^{s, t}(N \rtimes K)\right)$

$$
=(-1)^{s+t} \mid \operatorname{dim}_{\mathbb{C}}\left(H_{D}^{s, t}(N \rtimes K) \mid,\right.
$$

for $s, t \geq 0$, and where

$$
h_{D}(N \rtimes K)=\sum_{s, t} h_{D}^{s, t}(N \rtimes K) .
$$

The following is the main result of this section. For the case of $G$ finite, see Wang and Zhou [26, Theorem 3.1]. 
Theorem 6.4 Let $M$ be a compact, complex manifold on which the compact, complex, connected Lie group $G$ acts effectively, holomorphically and locally freely with integer shifts. The shifted delocalized orbifold Hodge polynomials satisfy

$$
\begin{aligned}
& \sum_{n \geq 0} h_{\mathrm{CR}}\left(M G\left(\mathcal{S}_{n}\right) ;-x,-y\right) q^{n} \\
&=\prod_{n=1}^{\infty} \prod_{s, t \geq 0} \frac{1}{\left(1-x^{s} y^{t} q^{n}(x y)^{(r-1) d / 2}\right)^{(-1)^{s+t} h_{\mathrm{CR}}^{s, t}(M \rtimes G)}} .
\end{aligned}
$$

Proof The result follows from Proposition 6.3 adjusted for the appropriate degree shift as in Zhou [29, page 5], together with Lemma 6.5 below, which can be proved by using local coordinates; see [26, Equation (9)].

Lemma 6.5 Let $M$ be a compact, complex manifold on which the compact, complex, connected Lie group $G$ acts effectively, holomorphically and locally freely with integer shifts. Then the degree shifts of the wreath product orbifold $M G\left(\mathcal{S}_{n}\right)$ are given by

$$
F_{\rho}=\sum_{r=1}^{n} \sum_{(c) \in t_{M} ; G} \sum_{j=1}^{N_{c}} m_{r,(c)}(j)\left(F_{j}^{c}+d \frac{r-1}{2}\right),
$$

where $\rho=\left\{m_{r}(c)\right\}_{r \geq 1,(c) \in t_{M ; G}}, m_{r}(c)=\sum_{j=1}^{N_{c}} m_{r,(c)}(j)$ and $\sum_{r,(c)} r m_{r}(c)=n$.

\section{References}

[1] A Adem, J Leida, Y Ruan, Orbifolds and stringy topology, Cambridge Tracts in Math. 171, Cambridge Univ. Press (2007) MR2359514

[2] A Adem, Y Ruan, Twisted orbifold K-theory, Comm. Math. Phys. 237 (2003) 533556 MR1993337

[3] M Atiyah, G Segal, On equivariant Euler characteristics, J. Geom. Phys. 6 (1989) 671-677 MR1076708

[4] B Blackadar, $K$-theory for operator algebras, second edition, MSRI Publ. 5, Cambridge Univ. Press (1998) MR1656031

[5] J Bryan, J Fulman, Orbifold Euler characteristics and the number of commuting $m$-tuples in the symmetric groups, Ann. Comb. 2 (1998) 1-6 MR1682916

[6] W Chen, Y Ruan, Orbifold Gromov-Witten theory, from: "Orbifolds in mathematics and physics (Madison, WI, 2001)", (A Adem, J Morava, Y Ruan, editors), Contemp. Math. 310, Amer. Math. Soc. (2002) 25-85 MR1950941

[7] L Dixon, J A Harvey, C Vafa, E Witten, Strings on orbifolds, Nuclear Phys. B 261 (1985) 678-686 MR818423 
[8] C Farsi, C Seaton, Generalized twisted sectors of orbifolds, Pacific J. Math. 246 (2010) 49-74 MR2645879

[9] C Farsi, C Seaton, Nonvanishing vector fields on orbifolds, Trans. Amer. Math. Soc. 362 (2010) 509-535 MR2550162

[10] F Hirzebruch, T Höfer, On the Euler number of an orbifold, Math. Ann. 286 (1990) 255-260 MR1032933

[11] S Illman, The equivariant triangulation theorem for actions of compact Lie groups, Math. Ann. 262 (1983) 487-501 MR696520

[12] K Kawakubo, The theory of transformation groups, Japanese edition, The Clarendon Press, Oxford Univ. Press, New York (1991) MR1150492

[13] I G Macdonald, The Poincaré polynomial of a symmetric product, Proc. Cambridge Philos. Soc. 58 (1962) 563-568 MR0143204

[14] I Moerdijk, Orbifolds as groupoids: an introduction, from: "Orbifolds in mathematics and physics (Madison, WI, 2001)”, (A Adem, J Morava, Y Ruan, editors), Contemp. Math. 310, Amer. Math. Soc. (2002) 205-222 MR1950948

[15] I Moerdijk, J Mrčun, Introduction to foliations and Lie groupoids, Cambridge Studies in Advanced Math. 91, Cambridge Univ. Press (2003) MR2012261

[16] I Moerdijk, D A Pronk, Simplicial cohomology of orbifolds, Indag. Math. (N.S.) 10 (1999) 269-293 MR1816220

[17] S-S Roan, Minimal resolutions of Gorenstein orbifolds in dimension three, Topology 35 (1996) 489-508 MR1380512

[18] Y Ruan, Stringy geometry and topology of orbifolds, from: "Symposium in Honor of C H Clemens (Salt Lake City, UT, 2000)”, (A Bertram, J A Carlson, H Kley, editors), Contemp. Math. 312, Amer. Math. Soc. (2002) 187-233 MR1941583

[19] I Satake, The Gauss-Bonnet theorem for V-manifolds, J. Math. Soc. Japan 9 (1957) 464-492 MR0095520

[20] C Seaton, Two Gauss-Bonnet and Poincaré-Hopf theorems for orbifolds with boundary, Differential Geom. Appl. 26 (2008) 42-51 MR2393971

[21] H Tamanoi, Generalized orbifold Euler characteristic of symmetric products and equivariant Morava K-theory, Algebr. Geom. Topol. 1 (2001) 115-141 MR1805937

[22] H Tamanoi, Generalized orbifold Euler characteristics of symmetric orbifolds and covering spaces, Algebr. Geom. Topol. 3 (2003) 791-856 MR1997338

[23] W P Thurston, The geometry and topology of three-manifolds, Princeton Univ. Math. Dept. Lecture Notes (1979) Available at http://msri.org/publications/books/ gt $3 \mathrm{~m} /$

[24] A Verona, Triangulation of stratified fibre bundles, Manuscripta Math. 30 (80) 425-445 MR567218 
[25] W Wang, Equivariant $K$-theory, wreath products, and Heisenberg algebra, Duke Math. J. 103 (2000) 1-23 MR1758236

[26] W Wang, J Zhou, Orbifold Hodge numbers of the wreath product orbifolds, J. Geom. Phys. 38 (2001) 152-169 MR1823666

[27] C T Yang, The triangulability of the orbit space of a differentiable transformation group, Bull. Amer. Math. Soc. 69 (1963) 405-408 MR0146291

[28] E Zaslow, Topological orbifold models and quantum cohomology rings, Comm. Math. Phys. 156 (1993) 301-331 MR1233848

[29] J Zhou, Delocalized equivariant coholomogy of symmetric products arXiv: math/9910028

Department of Mathematics, University of Colorado at Boulder

Campus Box 395, Boulder CO 80309-0395, USA

Department of Mathematics and Computer Science, Rhodes College

2000 North Parkway, Memphis TN 38112-1690, USA

farsi@euclid.colorado.edu, seatonc@rhodes.edu

http://www. colorado.edu/math/people/professors/farsi.html, http://faculty.rhodes.edu/seaton/

Received: 11 December 2009 Revised: 3 December 2010 